\title{
Integration of a 3D Variational data assimilation scheme with a coastal area morphodynamic model of Morecambe Bay
}

Article

Accepted Version

Thornhill, G. D., Mason, D. C., Dance, S. L., Lawless, A. S., Nichols, N. K. and Forbes, H. R. (2012) Integration of a 3D Variational data assimilation scheme with a coastal area morphodynamic model of Morecambe Bay. Coastal Engineering, 69. pp. 82-96. ISSN 0378-3839 doi: https://doi.org/10.1016/j.coastaleng.2012.05.010 Available at https://centaur.reading.ac.uk/28251/

It is advisable to refer to the publisher's version if you intend to cite from the work. See Guidance on citing.

To link to this article DOI: http://dx.doi.org/10.1016/j.coastaleng.2012.05.010

Publisher: Elsevier

All outputs in CentAUR are protected by Intellectual Property Rights law, including copyright law. Copyright and IPR is retained by the creators or other copyright holders. Terms and conditions for use of this material are defined in the End User Agreement. 


\section{CentAUR}

Central Archive at the University of Reading

Reading's research outputs online 


\section{Elsevier Editorial System(tm) for Coastal Engineering Manuscript Draft}

\section{Manuscript Number:}

Title: Integration of a 3D Variational data assimilation scheme with a coastal area morphodynamic model of Morecambe Bay

Article Type: Research Paper

Keywords: Coastal morphology; Morphodynamic modelling; Data assimilation; 3D-Var; Morecambe Bay

Corresponding Author: Dr Gillian Thornhill,

Corresponding Author's Institution: University of Reading

First Author: Gillian Thornhill

Order of Authors: Gillian Thornhill; David C Mason; Sarah L Dance; Amos S Lawless; Nancy K Nichols; Heather R Forbes

Abstract: This paper describes the implementation of a 3D variational (3D-Var) data assimilation scheme for a morphodynamic model applied to Morecambe Bay, UK. A simple decoupled hydrodynamic and sediment transport model is combined with a data assimilation scheme to investigate the ability of such methods to improve the accuracy of the predicted bathymetry. The inverse forecast error covariance matrix is modelled using a Laplacian approximation which is calibrated for the length scale parameter required. Calibration is also performed for the Soulsby-van Rijn sediment transport equations. The data used for assimilation purposes comprises waterlines derived from SAR imagery covering the entire period of the model run, and swath bathymetry data collected by a ship-borne survey for one date towards the end of the model run. A LiDAR survey of the entire bay carried out in November 2005 is used for validation purposes. The comparison of the predictive ability of the model alone with the model-forecast-assimilation system demonstrates that using data assimilation significantly improves the forecast skill. An investigation of the assimilation of the swath bathymetry as well as the waterlines demonstrates that the overall improvement is initially large, but decreases over time as the bathymetry evolves away from that observed by the survey. The result of combining the calibration runs into a pseudo-ensemble provides a higher skill score than for a single optimized model run. A brief comparison of the Optimal Interpolation assimilation method with the 3D-Var method shows that the two schemes give similar results. 
Environmental Systems Science Centre,

University of Reading,

Harry Pitt Building,

PO Box 238

Reading, RG6 6AL

UK

Email: G.D.Thornhill@reading.ac.uk

Phone: $+44(0) 1183787717$

Fax: $\quad+44(0) 1183786413$

Dear Sir/Madam,

I have submitted the paper titled 'Integration of a 3D Variational data assimilation scheme with a coastal area morphodynamic model of Morecambe Bay' for consideration for publication in the journal 'Coastal Engineering'.

The paper covers the application of $3 \mathrm{D}$ variational data assimilation to modelling of coastal bathymetry, and describes the improvement obtained in the predictions when this technique is used. This work extends the use of this method of data assimilation into a new area, and suggests ways in which near-shore bathymetry can be better modelled by using both data derived from SAR images and observations from a ship-based survey.

The work described has not been submitted for publication elsewhere, and we feel it is most suited to this journal.

Yours sincerely,

Gillian Thornhill

(Corresponding author). 
Integration of a 3D Variational data assimilation scheme with a coastal area morphodynamic model of Morecambe Bay

G. D. Thornhill ${ }^{1 *}$, D. C. Mason ${ }^{1}$, S. L. Dance ${ }^{2}$, A. S. Lawless ${ }^{2}$, N.K. Nichols ${ }^{2}$, H. R. Forbes ${ }^{1}$

${ }^{1}$ Environmental Systems Science Centre, University of Reading, Whiteknights, PO Box 238, Reading RG6 6AL, UK,

${ }^{2}$ Department of Mathematics and Statistics, University of Reading, Whiteknights, PO Box 220, Reading RG6 6AX, UK

*Corresponding author. Tel: +44 118 3787717; fax: +44 118 3786413; E-mail address:

G.D.Thornhill@reading.ac.uk (G. D. Thornhill)

\begin{abstract}
This paper describes the implementation of a $3 D$ variational (3D-Var) data assimilation scheme for a morphodynamic model applied to Morecambe Bay, UK. A simple decoupled hydrodynamic and sediment transport model is combined with a data assimilation scheme to investigate the ability of such methods to improve the accuracy of the predicted bathymetry. The inverse forecast error covariance matrix is modelled using a Laplacian approximation which is calibrated for the length scale parameter required. Calibration is also performed for the Soulsby-van Rijn sediment transport equations. The data used for assimilation purposes comprises waterlines derived from SAR imagery covering the entire period of the model run, and swath bathymetry data collected by a ship-borne survey for one date towards the end of the model run. A LiDAR survey of the entire bay carried out in November 2005 is used for validation purposes. The comparison of the predictive ability of the model alone with the model-forecast-assimilation system demonstrates that using data assimilation significantly improves the forecast skill. An investigation of the assimilation of the swath bathymetry as well as the waterlines demonstrates that the overall improvement is initially large, but decreases over time as the bathymetry evolves away from that observed by the survey. The result of combining the calibration runs into a pseudo-ensemble provides a higher skill score than for a single optimized model run. A brief comparison of the Optimal Interpolation assimilation method with the 3D-Var method shows that the two schemes give similar results.
\end{abstract}

Keywords: Coastal morphology; Morphodynamic modelling; Data assimilation; 3D-Var; Morecambe Bay 


\section{Introduction}

Understanding the processes acting in near-shore environments is increasingly important as the potential risk to coastal environments from the combination of sea-level rise and more frequent and intense storms resulting from global climate change needs to be assessed. In many coastal areas the local conditions can change rapidly, and tracking these changes is crucial for assessing flood risks, environmental impacts, safety for shipping and recreational uses of the coastal zones. In areas where the bathymetry evolves rapidly over time the collection of data from surveys and in-situ measurements with the frequency required to monitor the changes is not feasible due to cost and logistical difficulties. Newer approaches have used remote sensing to provide data over a larger area than local surveys typically provide, and allow the overall changes in local near-shore and inter-tidal bathymetry to be measured over the time-frame of the observations (e.g. Mason et al., 2010). In order to compensate for the general scarcity of data, complex computational morphodynamic models have been developed, including 3D morphodynamic models (e.g. Lesser et al., 2004). However, the ability of these models to predict the state of the near shore environment can be limited (de Vriend et al., 1993; Sutherland et al., 2004b) due to inadequate modelling of all the hydrodynamic processes acting, and imperfect knowledge of the initial conditions. The integration of remotely-sensed data with morphodynamic models offers the possibility to make predictions which are more accurate than using the models alone, and provide more insight into the evolution of the near-shore zones than intermittent data collection alone. Previous work using data assimilation to improve bathymetric modelling includes the Beach Wizard assimilation system, which used a combination of wave roller dissipation and inter-tidal bathymetry derived from video sources and radar-derived wave celerity as data sources. These were incorporated into the model using an optimal least squares estimator to update the model (van Dongeren, 2008), which resulted in improved skill for their predictions of near-shore bathymetry.

The work described here follows from the previous work of Scott and Mason (2007), where a simple coastal area morphodynamic model was coupled with an Optimal Interpolation (O.I.) data assimilation scheme. The objective of the current work is to implement a more powerful 3D variational (3D-Var) data assimilation scheme for a similar morphodynamic model, and to calibrate the system to produce the optimal results. The use of the 3D-Var method is widespread in operational data assimilation schemes in the Earth sciences because it is conceptually straightforward, computationally efficient for large problems, and can easily accommodate complex relationships between the observations and the model where the observed variable is not directly represented in the model. The 3D-Var method implemented here allows us to combine datasets of partial bathymetry from multiple sources in the assimilation scheme, and could be extended to investigate techniques for model parameter estimation in future (Smith et al., 2009). A comprehensive set of data consisting of SAR images, swath bathymetry and airborne LiDAR is available for the study site, Morecambe Bay in northwest England. The availability of independent datasets which can be used for model calibration, assimilation, and validation is an advantage over Scott and Mason (2007), where the data were limited to waterlines, and no independent verification data were available.

The study site is described in section 2 , and the data used for calibration, assimilation and validation is described in section 3 . In section 4 the data assimilation scheme used is introduced, with a discussion of the hydrodynamic and sediment transport model in section 5. Calibration of the model and assimilation scheme is presented in section 6 , and the results are given in section 7 . Section 8 compares the current 3D-Var assimilation implementation with the optimal interpolation method used in previous work; finally, conclusions and discussion are presented in section 9.

\section{Study Site - Morecambe Bay}

Morecambe Bay (Fig. 1) is a macrotidal embayment containing the largest single area of inter-tidal zone in Britain $\left(340 \mathrm{~km}^{2}\right)$. The extensive zone is in most part comprised of large sub-tidal channels and inter-tidal sandbanks, which can change in position and characteristics over very short periods of time. The Bay is fed by four main rivers, the Kent and Leven in the north and Lune and Wyre in the south. 
The ordinary spring tidal range is c. $8.2 \mathrm{~m}$, although the tidal range varies with location in the bay due to its size. The tidal currents are the dominant factor in sediment movement, as the landmasses of Ireland and the Isle of Man and the spits at the mouth of the bay limit wave size by restricting fetch (Mason et al., 1999). The duration of the semi-diurnal flood and ebb tides are unequal, with the ebb running for about 40 min longer than the flood at Heysham (Coomber and Hansom 1994). The spring tide attains a maximum velocity of $1.5 \mathrm{~ms}^{-1}$ in the large sub-tidal channels, with currents being higher on the flood than the ebb. The sediments in the inter-tidal zone are predominantly composed of very fine and fine sand $(0.06-0.2 \mathrm{~mm})$, with coarser sand and fine gravel at the mouth of the bay and silts in the inner bay (SMP, 1996). The channels and sandbanks in the bay constantly migrate and in the case of the Ulverston Channel there is evidence for migration of the order of $5 \mathrm{~km}$ over a 13 year period (Mason et al., 2010). Waves that propagate up the sub-tidal channels render some coastal areas vulnerable to flooding during storms, thus knowledge of the position of the channels and their evolution over time is essential to assessing the impact of future storms on coastal developments. The bay also contains a Special Area of Conservation and two Special Protection Areas for over-wintering birds, so the area has multiple issues for coastal management and protection. However, full surveys of the bathymetry of the bay are expensive to undertake frequently because of its large area, and improving the knowledge of the evolution of the bay will rely on combining theoretical models and partial observations derived from in-situ measurements and where available, from appropriate satellite and airborne imagery data. The availability of multiple datasets of the bay, the existence of a simple hydrodynamic model based on the tidal flow together with the dynamic nature of the inter-tidal channels made this an ideal study site.

(Figure 1).

\section{Datasets}

An extensive data set of inter-tidal bathymetry for Morecambe Bay was assembled for the study, including SAR images, swath bathymetry, and LiDAR, covering the period from January 2003 to November 2005. The SAR data are used to extract waterlines and provide height data for assimilation; the swath data are used for calibration purposes, and are also included in the assimilation for most of the results. The LiDAR data from November 2005 are used as a final validation dataset.

\subsection{Waterline data}

\begin{tabular}{|ccc|}
\hline $\begin{array}{l}\text { Table } 1 \\
\text { Waterline data used for assimilation }\end{array}$ & & \\
\hline Date (dd/mm/yy) & Time & Satellite \\
\hline $11 / 05 / 03$ & $11: 10: 00$ AM & ERS-2 \\
\hline $03 / 06 / 03$ & $10: 11: 00$ PM & ERS-2 \\
\hline $19 / 06 / 03$ & $09: 40: 00$ PM & ASAR \\
\hline $20 / 07 / 03$ & $11: 10: 00$ AM & ERS-2 \\
\hline $05 / 08 / 03$ & $11: 07: 00$ AM & ERS-2 \\
\hline $14 / 10 / 03$ & $11: 07: 00$ AM & ERS-2 \\
\hline $18 / 11 / 03 a$ & $10: 38: 00$ AM & ASAR \\
\hline $18 / 11 / 03 b$ & $11: 07: 00$ AM & ERS-2 \\
\hline $30 / 12 / 03$ & $10: 19: 00$ AM & ASAR AP VV \\
\hline $11 / 01 / 04$ & $11: 10: 00$ AM & ERS-2 \\
\hline $30 / 01 / 04$ & $10: 44: 00$ AM & ASAR(HH) \\
\hline $28 / 02 / 04$ & $10: 33: 00$ AM & ASAR AP HH \\
\hline $29 / 04 / 04$ & $09: 40: 00$ PM & ASAR \\
\hline $08 / 05 / 04$ & $09: 57: 00$ PM & ASAR AP VV \\
\hline $11 / 05 / 04$ & $11: 07: 00$ AM & ERS-2 \\
\hline $03 / 09 / 04 b$ & $10: 25: 00$ AM & ASAR \\
\hline
\end{tabular}




\begin{tabular}{|ccc|}
\hline $03 / 09 / 04 \mathrm{a}$ & $09: 49: 00 \mathrm{PM}$ & ASAR \\
\hline $14 / 12 / 04$ & $09: 43: 00 \mathrm{PM}$ & ASAR \\
\hline $29 / 06 / 05$ & $09: 52: 00 \mathrm{PM}$ & ASAR \\
\hline $28 / 08 / 05$ & $10: 42: 00 \mathrm{AM}$ & ASAR \\
\hline $22 / 11 / 05$ & $10: 39: 00 \mathrm{AM}$ & ASAR \\
\hline
\end{tabular}

The data used for the assimilation scheme is a set of waterlines extracted from SAR images following the procedure outlined in Mason et al. (1999) and Scott and Mason (2007). The term waterline is used to denote the water's edge, which moves to and fro as the tides rise and fall. A SAR image gives the position of the water's edge at the time of image acquisition; then the height of the water along the boundary (relative to a datum point) is found by running a hydrodynamic model including tide and surge components and interpolating the water elevations this predicts to the observed boundary. The bathymetry along the boundary is by definition equal to the water height, as the water depth along the boundary is zero. An example of a waterline is shown in Fig. 2(a). The data used and the dates on which they were acquired are given in Table 1. Each waterline is effectively a single irregular line running across the bay, and the position of the bathymetry from each waterline depends on the tide and surge conditions when the SAR image was acquired. Thus they are partial observations of the bay's inter-tidal bathymetry, and do not provide observations across the whole of the inter-tidal zone. They are also irregular in terms of the temporal resolution, in some cases there are only a few hours between waterline observations, and in other cases a gap of up to 6 months may occur.

(Figure 2)

\subsection{Swath data}

A bathymetric survey was carried out on Morecambe Bay in February 2005 by personnel from HR Wallingford, using an echo sounder aboard a survey vessel. The soundings were carried out along survey lines at $500 \mathrm{~m}$ spacings to define the low water channels. The area of the bay covered is shown in Fig. 2(b), where the black stripes represent where the soundings were taken. This dataset is more extensive spatially than a waterline, but represents a single point in time. These data are used in this work both as a calibration dataset, and also as a test of assimilation of data from different sources. This dataset is subsequently referred to as the swath bathymetry.

\subsection{LiDAR data}

These data (Fig. 2(c)) were obtained by Lancaster County Council in November 2005 from a scanning laser altimetry (LiDAR) survey that covered the entire inter-tidal zone of the bay. The spatial resolution was $2 \mathrm{~m}$, and the error on the mean heights was found to be $\pm 5 \mathrm{~cm}$ when compared with independently measured heights in urban areas around the bay. The complete data set included almost 200 million height samples. Such surveys are rare due to the difficulty of over-flying the bay at low tide, and the cost of acquiring and processing the data. These data were used solely for validation purposes in this study.

\section{Data Assimilation}

Data assimilation is a technique widely used in environmental science to combine information from a variety of sources of observational data with dynamic models. The purpose of this is to improve a model's predictive ability, and to permit a rigorous assessment of the relative accuracy of the model and the observations. A forecast is produced (referred to as the 'background') by running the model to the point where observations are available, then observations valid at the simulated time of the forecast are assimilated to produce a new model state called the 'analysis'. This is then fed back into the model, which continues to run until the next set of observations is available, and the process is repeated. The combination of the observations with the model prediction should produce an updated prediction that will be closer to the 'true' state than that of the model used alone.

It should be noted that the observations may be sparse, in the sense they may cover only a small portion of the model domain, and observations may not be available at regular intervals in time. In most assimilation schemes, the relative weighting of the background forecast and the observations is determined by a statistical estimate of the uncertainties in the model and observation data. The model 
and observation errors are often assumed to have Gaussian probability densities, with mean errors assumed equal to zero (if necessary bias correction can be carried out in a pre-processing step). The error covariance models play a crucial role in the assimilation (e.g. Bannister, 2008).

\subsection{D Variational data assimilation scheme}

There are various methods of data assimilation available to researchers (Bouttier and Courtier, 1999), and in previous work on data assimilation in Morecambe Bay an optimal interpolation (O.I.) scheme was used (Scott and Mason, 2007).

In this work a 3D variational assimilation scheme is adopted instead, as it is more efficient and can update the entire domain at once. In the case of O.I. the domain is usually broken down into smaller areas for the assimilation procedure, and the separate analyses are then combined to give the total solution. This can lead to unphysical solutions (Lorenc, 1986). It is also easier to incorporate observations from multiple data sources in 3D-Var, including indirect observations.

Defining the background state vector, which is effectively the model prediction, $z_{b}$, and assuming that this differs from the true state, $z_{t}$, by some amount $\eta$ which represents the vector of the estimation errors, we have:

$$
Z_{b}=Z_{t}+\eta
$$

As these errors are generally unknown, they can be modelled using the assumption of a Gaussian exponential function with a mean of zero.

The observations, $y$, may be irregularly spaced or of a variable not represented in the background vector. In order to compare the values of the observations with the state vector, an observation operator $\mathbf{h}$ is introduced. This may just be an interpolation from the model grid points to the observation points, or may include a conversion from the model variable to the observation variable and can be a non-linear function in the general case. As the observations will have associated errors $\varepsilon$ we can write this as

$$
y=\mathbf{h}\left(\mathbf{z}_{\mathrm{t}}\right)+\varepsilon,
$$

Similarly, we can treat these observation errors statistically, and can assess the errors on our data given the instruments and the methods used to obtain the observations.

The basis of 3D-Var methods is to formulate a cost function, which is a measure of the misfit between the model state and the observations and initial state:

$$
\mathbf{J}(\mathrm{z})=\left(\mathrm{z}-\mathrm{z}_{\mathrm{b}}\right)^{\top} \mathbf{B}^{-1}\left(\mathrm{z}-\mathrm{z}_{\mathrm{b}}\right)+(\mathrm{y}-\mathbf{H}(\mathrm{z}))^{\top} \mathbf{R}^{-1}(\mathrm{y}-\mathbf{H}(\mathrm{z}))
$$

where:
$\mathrm{z}$ is the model state vector (dimension $\mathrm{n}$, where $\mathrm{n}$ is the number of model grid cells)
$z_{b}$ is the background state vector (dimension $n$ )
$y$ is the observation vector (dimension $p$, with $p<<n$ )
$\mathbf{H}$ is the observation operator that maps model variables to observation space (in this case a simple linear interpolation from the model grid to the locations of the observations) (dimension $p$ $x \mathrm{n})$
B is the covariance matrix of background errors (dimension $n \times n$ )
$\mathbf{R}$ is the covariance matrix of observation errors (dimension $p \times p$ )

This cost function is minimized with respect to $z$, and the vector $z_{a}$ where the function is a minimum is referred to as the 'analysis'. The vector $z_{b}$ is the background vector, representing the bathymetry predicted by the model. The vector $z$ is usually taken to be the same as the background vector for the first iteration. In our case the observation operator $\mathbf{H}$ simply interpolates nearby model bathymetry values to the observation position. The matrix $\mathbf{B}$ is an error covariance matrix which represents the errors in model prediction (also referred to as the 'background' or 'forecast' errors), and $\mathbf{R}$ is the error 
covariance matrix for the observation errors. Choosing appropriate values and structures for these covariance matrices is important for the correct weighting between the observations and the background. In our implementation, the minimisation is done using a conjugate gradient method (Shanno and Phua, 1980), and the resulting analysis vector (the new bathymetry) is then used as the initial conditions for the next forecast of the bathymetric component of the model.

\subsection{The background covariance matrix $\boldsymbol{B}$}

Specifying the matrix $\mathbf{B}$ for a data assimilation scheme is fundamental to the correct working of the scheme, but being able to correctly assess the errors can prove difficult (Bannister, 2008). For our work we used an approximation for the error covariances that gave a tractable way to specify and manipulate the cost function.

The background error covariance can be written as $\mathbf{B}=\rho \sigma_{b}{ }^{2}$, where $\rho$ is a symmetric matrix of error correlations, and $\sigma_{b}{ }^{2}$ is the background error variance. The correlations are between the grid points corresponding to our single model variable representing the bathymetry, which will have a coefficient of 1 along the diagonal. Although $\rho$ is sometimes modelled using a continuous correlation function such as a Gaussian function, evaluated at the discrete grid-points (e.g., Smith, 2010) in the implementation of the 3D-Var scheme we need to have the inverse of the $\mathbf{B}$ matrix available. In our case the model has 34,000 grid cells, so the matrix B would have dimensions $34,000 \times 34,000$, which would be prohibitively expensive in computer time and resources to invert. Instead we have followed the work of (Johnson 2003, Johnson et al., 2005) and chosen to directly specify the inverse of the B matrix, using a Laplacian approximation as follows:

$$
\rho^{-1}=\gamma\left(\mathbf{I}+\left(\ell^{4} / 2\right) \mathbf{L}^{2}\right) / \ell
$$

where

$\gamma$ is a scaling factor

$\ell$ is the covariance length scale

$\mathrm{I}$ is the $\mathrm{n} \times \mathrm{n}$ identity matrix

$L$ is a 5 point discrete Laplacian operator of the form $1,1,-4,1,1$

This formulation results in a penta-diagonal matrix, which simplifies the matrix operations that need to be performed to calculate the cost function above. The scaling factor must be calculated such that the maximum element of $\rho$ is 1 . Note that this factor will change as the length scale is changed, but the value of $\gamma$ is independent of the dimensions of the matrix.

\begin{tabular}{|cc|}
\hline \multicolumn{2}{|l|}{$\begin{array}{l}\text { Table } 2 \\
\text { Values of } \gamma \text { for different length scales } \boldsymbol{\ell}\end{array}$} \\
\hline$\ell$ & $\gamma$ \\
\hline 0.5 & 0.335 \\
\hline 1 & 0.2131 \\
\hline 1.5 & 0.1356 \\
\hline 2 & 0.0981 \\
\hline 3 & 0.0629 \\
\hline 5 & 0.0362 \\
\hline
\end{tabular}

Using a test matrix of $25 \times 25$, we have calculated the value of $\gamma$ for several different length scales, shown in Table 2, where $\ell$ is defined as a multiple of the grid cell. This approximates the Gaussian correlation function, commonly used in defining the covariance matrix $\mathbf{B}$. This can be seen by comparing the result of the Gaussian-based correlation matrix to the inverse of $\rho^{-1}$ (i.e. $\rho$ ) produced by the Laplacian formulation (Johnson, 2003). Given the computational advantages of using the Laplacian form, we implemented this as the choice for defining directly the inverse covariance matrix $\mathbf{B}^{-1}$. 
The model background error variance $\sigma_{b}{ }^{2}$ is estimated by assuming that the variance of the difference between the waterline observations and the predicted model bathymetry at the time of the waterlines is the sum of the background and observation error variances at that time. These variances range from 0.73 to $3.34 \mathrm{~m}^{2}$. The overall observation variance is assumed to be $0.2 \mathrm{~m}^{2}$, as discussed in section 4.3, and subtracting this from the overall variance suggests a background variance of between 0.53 and $3.14 \mathrm{~m}^{2}$. Given this range, a value of $2.0 \mathrm{~m}^{2}$ was taken as a reasonable estimate for the background error variance for the assimilation runs. A test using a lower value for the background error variance is discussed in Section 6.4.

\subsection{The Observation Covariance matrix $\boldsymbol{R}$}

The observation error covariance matrix $\mathbf{R}$ was simplified to have a diagonal form, effectively assuming that there were no cross-correlations between the observation errors. A similar assumption is often made in numerical weather prediction, and allows the model to be more computationally efficient (Stewart et al., 2008, Stewart, 2010). There may be some error correlations between adjacent height samples along a waterline, though the waterlines were thinned to retain only the observation nearest the centre of each grid cell. However, here we are interested in the cross-correlations between the observation errors rather than the observations themselves. The calibration of the model waterline heights against the Heysham tidal gauge gave the same overall variance found by Scott and Mason (2007), and the same procedures were used for the waterline heighting, so the total errors estimated for this process was estimated to be $0.2 \mathrm{~m}^{2}$, the same as Scott and Mason (2007).

For the swath dataset, the measurement error was $0.0016 \mathrm{~m}^{2}$, while the dominant error was from the interpolation process, estimated to be $0.05 \mathrm{~m}^{2}$ as in Scott and Mason (2007). This value was taken as the variance for the swath observation in the assimilation process.

\subsection{Implementation}

The assimilation process was implemented by calculating the cost function of equation (3), using the current background bathymetry as the initial guess for the state of the bathymetry. The cost function was minimized using the method of conjugate gradients (Shanno and Phua, 1980). As an internal check on the consistency of the calculation, a 'gradient test' was applied (Jarvinen, 1998). This calculates two versions of the cost function separated by a small change in the model state, and compares this to the gradient of the cost function. Both should asymptotically point in the same direction, and failure to do so implies an error in the coding of the calculation, or in the assimilation system itself. This gradient test was applied to each assimilation, and provided an initial diagnostic that the assimilation system is working correctly.

\section{Description of Model}

\subsection{General outline of model}

The model used is described in detail in Scott and Mason (2007), and consists of a 2DH (depthaveraged) hydrodynamic component predicting tidal flows developed by the Proudman Oceanographic Laboratory, and a sediment transport component developed by Scott and Mason (2007). The model has to predict the continuous mutual adjustment of water flow and bathymetry in the bay, as the water flow causes sediment transport, which changes the bathymetry, which alters the water flow, and so on. The model should ideally solve all the equations covering both the water flow and the sediment transport simultaneously, but to simplify the model numerics, the hydrodynamic and sediment transport components are decoupled. This allows the hydrodynamic component to be run while keeping a constant bathymetry, and then the bathymetric component to be updated prior to recalculating a revised water flow (de Vriend, 1987). However, it is important to maintain the correct feedback between the two parts of the model. This is done using a continuity correction, which corrects the depthaveraged currents and water heights from the hydrodynamic component output at 5 minute intervals assuming that the water height and flux are constant with changing bathymetry within the morphodynamic time-step.

This work is aimed at investigating the use of data assimilation in coastal area morphodynamic modelling rather than the development of a detailed and accurate model unaided by assimilation, so a relatively simple morphodynamic model is used. The model is run for a period of 23 months from a start point at January 2004 to November 2005. This covers the period during which the waterline data and swath bathymetry were acquired, and ends just after the time of the LiDAR overflight. The low resolution of the model domain compared to the data from the swath bathymetry 
required that the dataset was down-sampled to match the model grid cell size of $240 \mathrm{~m}$, averaging the data such that only one data point per model grid cell was used for the assimilation. The original waterline data was also similarly thinned to give a single value for each grid cell, following Scott and Mason (2007).

\subsubsection{Hydrodynamic component of the model}

The hydrodynamic part of the model predicts the water flow due to the tides, with the surge component being ignored (though surge is employed in calculating heights for individual waterlines). The model also does not include wave action. The time step used is $4 \mathrm{~s}$, and the spatial resolution is $240 \mathrm{~m}$, which results in a grid size of 196 X 176 cells for the entire domain. It takes the bathymetry, the depthaveraged current and water height, and the tidal forcing along the seaward edge of the bay as inputs as described in Mason 1999), and produces updated water heights and depth-averaged currents, which are then used as input for the sediment transport model and bathymetric component. The model provides this output at intervals of 5 minutes, considered to be adequate for the tidal conditions without requiring excessive transfer of data to the bathymetric component.

\subsubsection{Sediment transport component of the model}

The sediment transport component uses the same model grid as the hydrodynamic component, and takes the water depth, depth-averaged current and initial bathymetry as the basis for calculating the sediment transport and resulting new bathymetry. It solves the sediment conservation equation using a Lax-Wendroff numerical scheme to solve the equation in the advection-form. The details of the model and the solution scheme are provided in Scott and Mason (2007), however in this work the sediment transport rate is calculated using a simplified form of the Soulsby-van Rijn equation instead of the power law equation. The contribution from waves has been shown to be small (Mason and Garg, 2001) and was excluded from the model.

The sediment transport equation is given by

$$
q=A_{s} u\left[|u|-\left|u_{c r}\right|\right]^{2.4}
$$

where

$$
\begin{aligned}
& \mathrm{A}_{\mathrm{s}}=\left(\mathrm{A}_{\mathrm{ss}}+\mathrm{A}_{\mathrm{sb}}\right) \mathrm{F}, \\
& A_{s s}=\frac{0.012 d_{50}-D^{-0.6}}{\left[(s-1) g d_{50}\right]^{1.2}}, \\
& A_{s b}=\frac{0.005 h\left(d_{50} / h\right)^{1.2}}{\left[(s-1) g d_{50}\right]^{1.2}}, \\
& D=\left[\frac{g(s-1)}{v^{2}}\right]^{1 / 3} d_{50},
\end{aligned}
$$

$$
\begin{array}{ll}
\mathrm{A}_{\mathrm{ss}} & =\text { suspended sediment load } \\
\mathrm{A}_{\mathrm{sb}} & =\text { bed sediment load } \\
\mathrm{u} & =\text { depth-averaged current } \\
\mathrm{u}_{\mathrm{cr}} & =\text { critical current for sediment movement } \\
\mathrm{h} & =\text { water depth }
\end{array}
$$




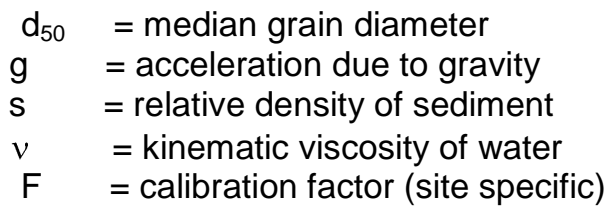

The median diameter $d_{50}$ was set at $0.12 \mathrm{~mm}$ (Scott and Mason, 2007), and was assumed constant across the bay. A scale factor $F$ was used as a multiplier to calibrate the sediment transport rate $q$ for site-specific conditions (Soulsby, 1997), as this formulation has been shown to under-estimate the sediment transport for fine-grained sand, such as exists in Morecambe Bay (Camenen and Larroude, 2003). The value for $F$ is assessed below as part of the model calibration procedure.

Following each time-step of the sediment transport component of the model, the hydrodynamic component should ideally be restarted with the new bathymetry to provide tidal data for the next timestep, but the continuity correction is considered to remain acceptable over the time scale of 1 hour, after which the hydrodynamic component is restarted using the new bathymetry. This short time step is the morphodynamic time-step, and reflects the fact that the bathymetry in Morecambe Bay can change significantly over a period of hours (Scott and Mason, 2007).

\subsubsection{Overview of model run sequence}

The sequence of events for the model run is: the hydrodynamic part is run for a period of $1 \mathrm{~h}$, and the currents and water heights are saved every 5 minutes. Then the sediment transport component is run for the same period, with the depth-averaged currents updated every 5 minutes with the continuity correction. The sediment transport rates are calculated, and a new bathymetry produced using the sediment conservation equations. At the end of the $1 \mathrm{~h}$ run, the bathymetry is stored and fed back to the hydrodynamic model, which continues for the next $1 \mathrm{~h}$ step.

\subsection{Initial bathymetry}

In general it may be difficult to provide a correct initial bathymetry in most operational cases and the most likely situation would be an imperfect or out-of-date bathymetry for the beginning of a model run. In this case, we wanted to provide the best estimate of the likely bathymetry at the start of our model run thus reducing the probable errors for the initial conditions and providing a more stringent test for the comparison of predictions with and without assimilation. The differences between the previously available bathymetry valid for 1997 (Mason et al., 1999) and the LiDAR data for November 2005 show that substantial changes in the channel positions had occurred over this period. In order to update the 1997 data to provide a new initial state for 2004, we ran the model using the earlier data and assimilated the waterlines available for May 2003 - December 2004. This was done using default parameters for the model and assimilation, and was the simplest method for updating the old bathymetry to a state which would be more likely to represent the 'true' state of the bathymetry for the beginning of our model runs in January 2004. Fig. 3(a) and (b) show the differences between the 1997 bathymetry and our updated initial bathymetry.

(Figure 3)

\section{Calibration of the Assimilation System}

The system needs to be calibrated for the sediment transport parameter $F$, and also to find the best value of the covariance length scale $\ell$ to produce the best results for the assimilation scheme. This was done by changing the values of the parameters over a reasonable range, and using performance measures to select the values providing the best predictions of the bathymetry.

\subsection{Performance measures for the calibration}

The calibration was carried out against the swath bathymetry from February 2005, as this covered a larger part of the domain than the individual waterlines. The model was run to the time of the swath dataset, and then the predicted bathymetry was compared to the swath bathymetry wherever they overlapped, using interpolation to match the model points to the data. Measures of model performance were calculated so that the impact of different values of model parameters could be assessed. The main measures calculated from the set of height differences were the mean difference, the Mean Square Error (MSE), the variance, and the Brier skill score (Sutherland et al., 2004a). The Mean 
Absolute Error (MAE) did not change substantially between model runs, and was not considered useful for discriminating between them. The Brier skill score is a measure of how much better the model matches the observed data compared to a baseline bathymetry (in our case the baseline is taken to be the initial bathymetry for January 2004), so that if the model produces bathymetry that differs from the observed bathymetry by more than the initial bathymetry, the skill score is negative. The Brier skill score is defined as

$$
\text { Brier Skill Score }=1-\frac{\left\langle\left(b_{m}-b_{o}\right)^{2}\right\rangle}{\left\langle\left(b_{i}-b_{o}\right)^{2}\right\rangle}
$$

where

$$
\begin{aligned}
& b_{o}=\text { vector containing observations of bathymetry } \\
& b_{m}=\text { vector containing modelled bathymetries for each observation in } b_{\circ} \\
& b_{i}=\text { vector containing initial bathymetries for each } b_{\circ} \\
& <>=\text { average over the observations }
\end{aligned}
$$

For a perfect prediction, the score would be 1.0. Although there are caveats to the use of this measure, for example when the denominator is small (Sutherland et al., 2004), it can still be used as a comparative estimator of skill between models with the same baseline bathymetry as in this work. Note that in the calibration section, the bathymetry observations are the swath data, and in the results, we calculated the skill score with respect to the LiDAR data. This means the two sets of scores are not directly comparable with each other, as the denominator will be depend on the observations used.

\subsection{Calibration of the covariance length scale}

The Laplacian structure of the $\mathbf{B}^{-1}$ matrix has a length scale $\ell$ that controls the distance over which the information from an observation is spread. To calibrate the best value for $\ell$, several runs of the assimilation system were made varying this parameter from 0.5 to 3.0 grid cells. The results in Fig. 4 show the difference in bathymetry between the background and the analysis (i.e. immediately before and after the assimilation) for the assimilation of the first waterline, which occurs after 10 days of model run time. In Fig. 4 (a) the value of $\ell$ is 0.5 , and the changes from the assimilation of the data are limited to the areas immediately around the waterline itself. Fig. 4(b) shows the same assimilation, but using a value of $\ell=3.0$ and demonstrates the additional spreading of the information from the observations for this value of $\ell$. The bathymetry is not smoothly varying, and can have relatively sharp gradients (channel sides, for example), which means that taking too large a value for $\ell$ will extend the updates from the observations beyond the region where they will be applicable. This can erroneously smooth over finer scale features in the bathymetry and could produce misleading results with channels being in the wrong place, or broadened out unrealistically. The choice of length scale here is tuned so that the geometry of the channels as outlined in the observations is preserved. If the length scale is too small, the observations will be confined to too small an area, and will not improve the overall bathymetry sufficiently. The difference in the predicted bathymetry (after a 23 month run) between a run using a value of $\ell$ of 0.5 and one using $\ell=3.0$ is shown in Fig. 5(a). The value of the length scale affects the way the observations are assimilated and therefore on the resulting bathymetry, and these effects are compounded after each assimilation over the model run period. The differences cover a large proportion of the model domain because $\ell$ is directly involved with the spatial distribution of the information contained in the observations. The position of the channels is affected quite strongly by the value of $\ell$, in particular the Ulverston Channel which appears as the large red and orange area (marked as $A$ in Fig 5(a)) showing where the larger value of $\ell$ has not constrained the observations tightly enough, and the channel migration is incorrectly represented. The performance measures were calculated for the runs over the range of $\ell$, and the results are shown in Table 3 . The Brier skill score, MSE and variances all deteriorate for values above $\ell=1.0$ which produced the best performance measures and was therefore used in subsequent runs of the assimilation scheme.

(Figure 4)

(Figure 5) 


\subsection{Calibration of sediment transport factor $F$}

The Soulsby-van Rijn equation (5) is used to calculate the sediment transport and includes a calibration factor $F$. Several runs of the model were carried out using different values of $F$, ranging from $F=0.1$ to $F=3.0$. The other parameters were held constant, with the length scale of the $\mathbf{B}^{-1}$ matrix set to 1.0 , and the value of $\sigma_{\mathrm{b}}{ }^{2}$ set to 2.0. The main impact of increasing $F$ is to increase the sediment transport for a given tidal flow, and the channels tend to be deeper for a larger value. This can be seen by comparing the bathymetry output for the lowest and highest value of $F$ in Fig. 5(b). This shows the difference in bathymetry after 23 months between two model runs, one using $F=0.1$ and one with $F=3.0$ with a positive difference where the values for $F=0.1$ are higher than those for $F=3.0$. Comparing Fig. $5(b)$ with Fig. 5(a), the effects of $F$ are less widespread as it has the greatest impact where erosion and deposition are occurring, and it is not affected directly by the assimilation. The shallower erosion of channels for the low value is clearly seen in the Ulverston channel and the lower reaches of the Channel leading up to the Kent Estuary (marked A and B on Fig 5(b)). Greater deposition is seen near Grange-over Sands and along the edges of Wharton Sands where the Kent Estuary joins the main part of the bay ( $C$ and $D$, respectively). The values of the performance measures are calculated and the results are shown in Table 3 . The best results from the calibration are for a value of $F=2.0$ have the optimal values, and this was used for the nominal runs presented in section 7 .

Table 3 Calibration results for values of $F, \ell$ and $\sigma_{b}^{2}$

\begin{tabular}{|c|c|c|c|c|c|c|}
\hline $\mathbf{F}$ & $\sigma_{b}^{2}$ & $\ell$ & $\begin{array}{c}\text { Mean } \\
\text { Differences } \\
(\mathrm{m})\end{array}$ & $\begin{array}{l}\text { MSE } \\
\left(\mathrm{m}^{2}\right)\end{array}$ & $\begin{array}{c}\text { Variances } \\
\left(\mathrm{m}^{2}\right)\end{array}$ & BSS \\
\hline 0.1 & 2.0 & 1.0 & -0.810 & 2.650 & 2.000 & 0.215 \\
\hline 0.5 & 2.0 & 1.0 & -0.760 & 2.660 & 2.090 & 0.211 \\
\hline 1.0 & 2.0 & 1.0 & -0.550 & 2.360 & 2.060 & 0.310 \\
\hline 1.5 & 2.0 & 1.0 & -0.330 & 2.160 & 2.060 & 0.360 \\
\hline 2.0 & 2.0 & 1.0 & -0.170 & 2.090 & 2.060 & 0.382 \\
\hline 3.0 & 2.0 & 1.0 & 0.030 & 2.540 & 2.540 & 0.248 \\
\hline 2.0 & 2.0 & 0.5 & -0.090 & 2.330 & 2.320 & 0.311 \\
\hline 2.0 & 2.0 & 1.0 & -0.170 & 2.090 & 2.060 & 0.382 \\
\hline 2.0 & 2.0 & 2.0 & -0.360 & 2.430 & 2.300 & 0.281 \\
\hline 2.0 & 2.0 & 3.0 & -0.400 & 2.770 & 2.610 & 0.181 \\
\hline 2.0 & 0.8 & 1.0 & -0.170 & 2.150 & 2.120 & 0.151 \\
\hline
\end{tabular}

\subsection{Impact of the background variance}

The value of the variance $\sigma_{b}{ }^{2}$ for the background errors was set to be $2.0 \mathrm{~m}^{2}$. However, in the previous work of Scott and Mason (2007), a value of $0.8 \mathrm{~m}^{2}$ was chosen. An examination of the impact of this value was undertaken, by comparing assimilation runs using both the waterline and swath data with each value. The other parameters values used for this comparison were $\mathrm{F}=2.0, \ell=1.0$.

The effect of increasing $\sigma_{b}{ }^{2}$ is to increase the value of the elements in the error covariance matrix for the model forecast, so a larger value would effectively increase the weight applied to the observations in the assimilation scheme. A plot of the differences in the bathymetry predicted using a value of $\sigma_{b}{ }^{2}=$ $0.8 \mathrm{~m}^{2}$ and a value of $\sigma_{\mathrm{b}}{ }^{2}=2.0 \mathrm{~m}^{2}$ after the 23 month model run is shown in Fig. 5(c) (note the scale bar in this case runs from $-2.0 \mathrm{~m}$ to $2.0 \mathrm{~m}$ ). It shows that the differences are not very large, and as expected are concentrated in areas where there the assimilation has made the most difference to the channel positions and morphology. Overall the bathymetry produced by the model is not very sensitive for these values of the parameter (Table 3).

\section{Results}

The results discussed in the following section examine the effects of the assimilation system in detail. The assimilation system was run in several configurations: (i) without assimilation, (ii) assimilating the waterlines and the swath bathymetry, and (iii) assimilating only the waterlines in order to assess how the system behaves without the addition of the swath survey data. We also produced a prediction of 
the bathymetry based on combining all the calibration results. All of the results were compared to the LiDAR data, and performance measures for the individual runs were calculated against this dataset.

\subsection{Impact of assimilation on the predicted bathymetry}

To show the impact of the assimilation, two 23-month runs are compared, one using the assimilation of the waterlines and the swath data, the other with no data assimilation. In both cases the value of $F=$ 2.0, and a scale length $\ell=1.0$ is used in the case where assimilation is used. The use of data assimilation provides a substantial improvement over the use of the model alone, as seen in Table 4 where the performance measures are compared. The mean differences, MSE and Brier skill scores all show a large improvement when assimilation is used. Plots of the differences between the resultant runs and the LiDAR data (prediction - data) are shown in Fig. 6(a) and (b). The run that included assimilation has fewer areas of difference with the LiDAR data, and where the differences exist they tend to be less in absolute terms than for the model run without assimilation.

The improvement provided by including the assimilation is most marked where the Ulverston Channel has moved over the course of the model run; in the non-assimilation run this channel has remained in its initial position, which can be seen in the large negative difference in Fig. 6(a) (marked A). The changes in the Kent and lower Lune estuaries (A and B in Fig 6(b)) also show some improvement in the assimilated runs. Overall the waterlines and swath bathymetry substantially reduce the differences between the LiDAR data and the model output, and improve the prediction for the bathymetry considerably. The values for the mean differences, MSE and Brier skill score for the two runs when compared with the LiDAR data at the end of the model run are shown in Table 4. The assimilation results in a great improvement for the model, reducing the errors substantially, and improving the Brier skill score to a value of 0.262 .

(Figure 6).

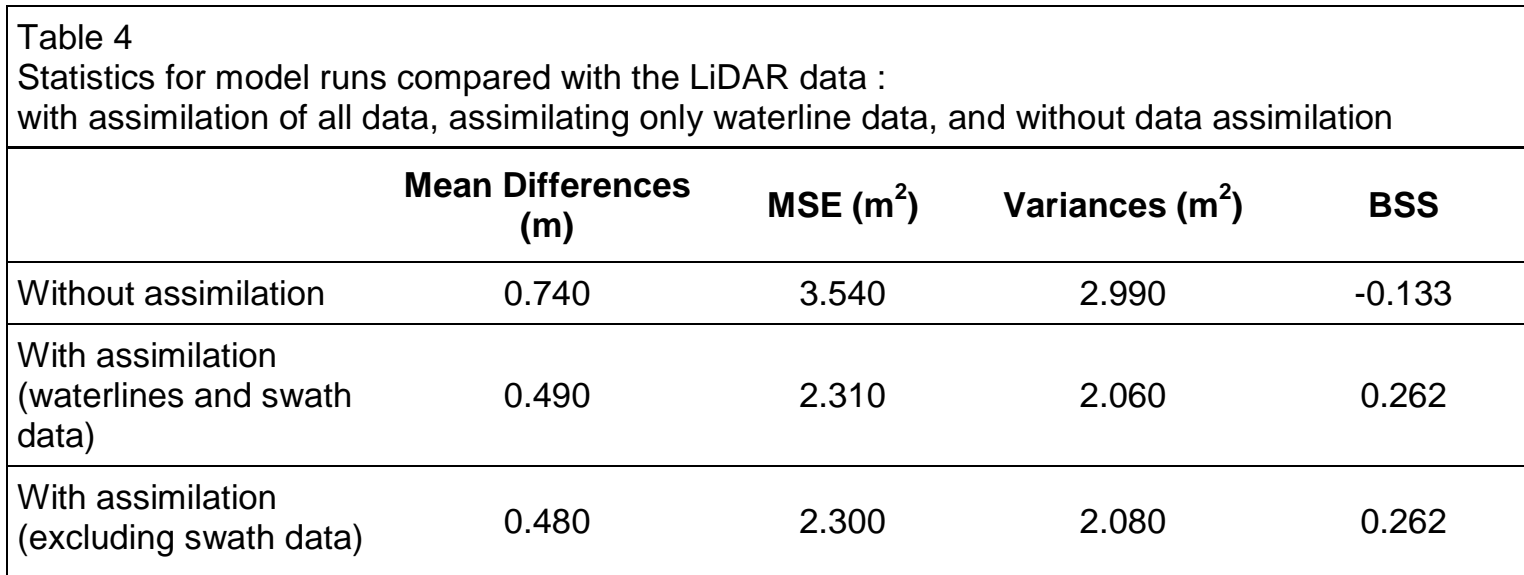

7.2 The impact of removing the swath bathymetry dataset from the assimilation

The inclusion of all data available for an assimilation system should maximise the effectiveness of the assimilation system. However, the additional swath data may not be available for other sites and to investigate the improvements provided by assimilating the waterlines only we ran some experiments excluding the swath data from the assimilation. Fig. 6(c) shows the differences between the predicted bathymetry and the LiDAR data when the swath data are excluded from the assimilation. Comparing this with Fig. 6(b) shows the influence of the assimilation of the swath data in improving some of the channel positions and depths although the effect is not as pronounced as might be expected. The values for the MSE and Brier skill scores calculated using the LiDAR validation data for the runs with and without the swath data are shown in Table 4 and show that there is almost no difference in the performance measures between the two runs. Although this implies that adding the swath data does not improve the model skill, this could be explained by the 9 month interval between the swath observations and the LiDAR observations. During this time it is likely that the bathymetry has changed sufficiently that the earlier swath data no longer represent an accurate picture of the bathymetry at the end of the model run. The swath data also mainly covers the lower inter-tidal zone and outside of this area there is less of an impact which may also affect the overall performance measures. To investigate the removal of the this data from the assimilation scheme in more detail we compared how the 
predicted bathymetry evolves between the time of the swath observations and the end of the model run for the cases with and without the assimilation of this dataset ('with swath' and 'without swath'). There are two waterline assimilations occurring between the times of the swath observations and the LiDAR data which will correct some of the discrepancies for the model during this period (a third waterline assimilation occurs just after the LiDAR observations were taken). In Fig. 7 the Brier Skill Scores for the predicted bathymetry are calculated using the swath data as the observations so indicate how different the predicted bathymetry is from this dataset, and are plotted as a function of time. In the case where the swath is assimilated, the bathymetry produced immediately afterwards has a very high Brier skill score as the new bathymetry has been adjusted using the same dataset as appears in the Brier skill score calculation, while the model run without the swath data has a much lower initial Brier skill score at this time. The skill score in both cases then decreases with time after the assimilation, as the predicted bathymetry drifts further from the swath data. However, it is interesting to note the effect of the next waterline assimilations (denoted on the graph by the lines marked 'WL'). The first waterline assimilation causes a marked reduction in the Brier skill score for the case including the swath data assimilation, indicating that there is a difference between the bathymetry along the waterline and the swath data in the 121 days between the two datasets. In the case where the swath data was not assimilated, and thus has not influenced the prediction, we also see a reduced skill score after this waterline assimilation; this suggests that the difference between the predicted bathymetry and the waterline dataset is not altogether due to the assimilation of the swath data. As this next assimilation occurs for both runs, the difference in the predicted bathymetry between them is being reduced. The 'with swath' Brier skill score remains relatively constant after that; however, in the case of the 'without swath' run, the skill score improves slightly, indicating that the predictions are becoming closer to the swath observations. For the next waterline assimilation, the change is negligible for the 'with swath' run, but there is a slight improvement for the 'without-swath' run; this implies that the observations from this waterline data are adjusting the bathymetry slightly closer to the swath data. The final waterline assimilation produces a similar improvement in both cases, although it should be noted that this assimilation occurs after the date of the LiDAR validation data. Although when compared with the LiDAR data the inclusion of the swath data does not appear to improve the predictions, this is most likely due to the changes in the bathymetry which occur after the date of the swath observations. The subsequent assimilation of waterlines then brings the bathymetry from both runs closer together, and reduces the differences produced by including the swath data.

(Figure 7)

\subsection{Combining multiple realizations as a pseudo-ensemble}

7.3.1 Parameter Ranges

The set of results obtained for the calibration of the assimilation system, covering ranges of the parameters $F, \ell$, and $\sigma_{b}{ }^{2}$, were taken together to constitute a form of ensemble. Although this ensemble is not produced by using a probability density function of the various perturbations as a formal ensemble might, it does give us a way of assessing the uncertainty of our final bathymetry prediction. This is done by combining the bathymetries from all the runs as a weighted average, and also producing a weighted standard deviation for the full set of parameter ranges for $F, \ell$, and $\sigma_{b}{ }^{2}$. The weights were obtained by calculating the inverse of the MSE for each run, normalising the sum of the inverses to one, and assigning weights based on the result (Woodcock and Engel, 2005).

The weighted average of these model bathymetries is then subtracted from the LiDAR bathymetry. A chi-squared statistic is calculated for the resulting 'uncertainty map' of the difference bathymetry using the standard equation for a reduced chi-squared test (Laub and Kuhl, 2005). This is done by taking the differences between the ensemble mean at each grid cell and the LiDAR data, to produce a set of 'observed' differences, $y_{i}$, and assuming that the predicted mean difference at each cell, $f\left(x_{i}\right)=0$ if the model is perfect, as follows :

$$
X_{\text {red }}^{2}=\frac{1}{v} \sum_{i} \frac{1}{\sigma_{i}^{2}} \mathbf{l}_{i}-f\left(x_{i}\right)_{-}^{2}
$$

where:

$$
\begin{aligned}
& y_{i}=\text { observed height difference at i'th grid cell } \\
& \sigma_{i}^{2}=\text { variance related to measurement error on } y_{i}
\end{aligned}
$$




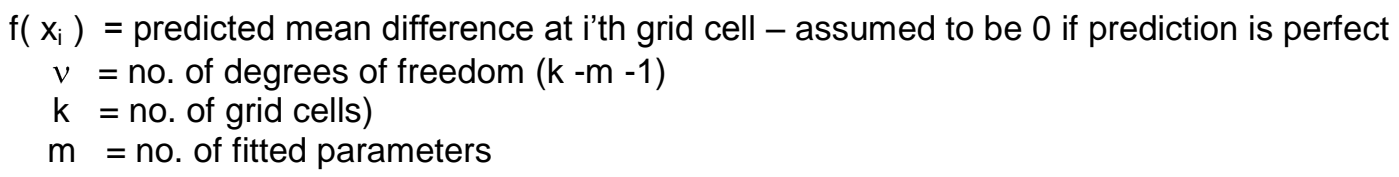

The advantage of the reduced chi-squared compared to the normal chi-squared test is that it normalizes for the number of degrees of freedom.

The variance $\left(\sigma_{1}{ }^{2}\right)$ at the i'th grid cell is taken as the weighted standard deviation of the model runs squared, added to the variance for the sub-sampled LiDAR observations $\left(0.05 \mathrm{~m}^{2}\right)$. This gives us the combined variance of the difference between the observation and the combined model runs at each grid cell. On the assumption that the null hypothesis is true, the values of the predicted differences $f\left(x_{i}\right)$ are zero. In principle, a reduced chi-squared value of 1 indicates that the extent of the match between observations and predictions is in accord with the error variance. However, the result obtained was a high $\mathrm{X}_{\text {red }}{ }^{2}$ value of 10.88 , as the model fit is not good in all areas of the domain. The performance measures for the individual runs used to construct the ensemble and the ensemble itself are given in Table 5 (note the dataset used for the observations in the Brier skill score is the LiDAR dataset). The results for the ensemble are considerably better than those for any of the individual runs; possibly because different parts of the bay have different sediment grain sizes and sediment transport for the same tidal regime may be variable, which would suggest a spatially variable value for $F$ might provide better results for single runs. The effect of the length scale could also improve results if it was variable; flatter areas of the inter-tidal regime could support a larger value, and a smaller value would be more appropriate where the bathymetry is changing rapidly across a few grid cells, e.g. at channel boundaries. To construct an uncertainty map for the ensemble bathymetry we calculated the weighted standard deviation for the ensemble compared to the LiDAR data (Fig. 8). This calculation includes the standard deviation for the observations, as this is included in the uncertainty of the predicted bathymetry compared to the true bathymetry. The areas with the largest uncertainty correspond to where the fit with the LiDAR data is least reliable, and tend to be where the varying the values of $F$ and $\ell$ have the greatest impact (c.f. Fig. 5). This gives us a representation of where the predicted bathymetry is most sensitive to the values of these parameters by combining them together, and providing an overall estimate of where the bathymetry may be less reliable due to uncertainties in the calibration, or biases caused by the inadequate spatial coverage of the calibration dataset.

(Figure 8)

\subsubsection{Bathymetry results from combined realizations.}

The performance measures for the ensemble are shown in Table 5, where the results for the ensemble provide an improvement over the best individual run, and reduce the differences between the validation data and the predicted bathymetry. This may be due to combining the parameter values so that there is effectively a variable value of the parameters from the different individual runs providing a better overall match. A plot showing the differences between the LiDAR data and the weighted mean of the runs (Fig. 6(d)) shows where the ensemble prediction has improved over the nominal run shown in Fig. 6(b). The ensemble still does not correctly predict the deposition at the northern edge of the Ulverston Channel, (A) although there is some improvement on the western branch of this channel (B). The area where the ensemble fails to predict the bathymetry are in the details of the channel positions; the modelling does not include the processes that lead to migration of the channels, and it is in these areas where we are most reliant on the data assimilation. If the observations do not cover these areas well, or are too sparse in time, we miss some of those changes. The deposition extending from Humphrey Head is not well captured, for example (C). This latter area is one where there are no observations over the entire period of the model run, so the correction to the model in this area will be weaker than elsewhere. Another region which is not well represented is the Lune Estuary (D), where the model has not captured the position of the channel well. Overall, the ensemble bathymetry has fewer differences from the LiDAR data compared to the nominal run, and this approach can provide improvements in the predictive capability of the assimilation system. The other advantage is that an uncertainty map can be constructed so that the reliability of the bathymetry can be assessed.

\subsection{Comparison of the predicted bathymetry with the LiDAR validation data}

The results from all the runs of the assimilation scheme were compared with the final LiDAR data, and the performance measures for this comparison are shown in Table 5. Using these values, the best 
Brier skill score is found for a value of $F=1.0$ and $\ell=1.0$. This contrasts with the original calibration against the swath data where a value of $F=2.0$ gave the better results (as shown in Table 3 ) for the equivalent parameter values. The discrepancy could be due to the LiDAR data covering the entire domain, including the higher reaches of the channels, while the swath data is limited to the main channels in the deeper areas of the inter-tidal zone. This may have biased the calibration using the swath data in favour of greater sediment transport with the higher value of $F$, which may be more appropriate to these areas of the bay. The sediment transport in the higher reaches of the bay may be better represented by a smaller value of $F$, but without independent calibration data covering these areas this could not be verified. In general, the Brier skill scores for the LiDAR data are slightly lower than the calibration skill scores, but the overall differences between the highest scores are not large (0.382 for the swath data, 0.328 for the LiDAR data).

\begin{tabular}{|c|c|c|c|c|c|c|}
\hline \multicolumn{7}{|c|}{$\begin{array}{l}\text { Table } 5 \\
\text { Performance measures for individual runs and the combined mean of all results compared } \\
\text { to the LiDAR data }\end{array}$} \\
\hline $\mathbf{F}$ & $\sigma_{b}^{2}$ & $\ell$ & $\begin{array}{l}\text { Mean } \\
\text { Differences } \\
(\mathrm{m})\end{array}$ & $\operatorname{MSE}\left(m^{2}\right)$ & $\begin{array}{l}\text { Variances } \\
\qquad\left(\mathrm{m}^{2}\right)\end{array}$ & BSS \\
\hline 0.1000 & 2.0 & 1.00 & 0.500 & 2.350 & 2.100 & 0.293 \\
\hline 0.5000 & 2.0 & 1.00 & 0.470 & 2.150 & 1.930 & 0.310 \\
\hline 1.0000 & 2.0 & 1.00 & 0.480 & 2.100 & 1.860 & 0.328 \\
\hline 1.5000 & 2.0 & 1.00 & 0.490 & 2.260 & 2.020 & 0.276 \\
\hline 2.0000 & 2.0 & 1.00 & 0.490 & 2.310 & 2.060 & 0.262 \\
\hline 3.0000 & 2.0 & 1.00 & 0.470 & 2.600 & 2.380 & 0.166 \\
\hline 2.0000 & 2.0 & 0.50 & 0.510 & 2.510 & 2.250 & 0.196 \\
\hline 2.0000 & 2.0 & 2.00 & 0.480 & 2.140 & 1.900 & 0.316 \\
\hline 2.0000 & 2.0 & 3.00 & 0.530 & 2.460 & 2.180 & 0.212 \\
\hline 2.0000 & 0.8 & 1.00 & 0.480 & 2.210 & 1.980 & 0.227 \\
\hline \multicolumn{3}{|c|}{ Mean of combined results } & 0.49 & 1.99 & 1.75 & 0.364 \\
\hline
\end{tabular}

\subsection{Predicted changes in bathymetry over model run period}

The changes in the bathymetry over the course of the 23 month run shown in Fig. 9(a) are based on comparing the initial bathymetry from January 2004 used for the model run, and the bathymetry as measured by the LiDAR data in November 2005. The LiDAR data are averaged to produce a single point for each model grid cell for this comparison and this is subtracted from the initial bathymetry. This is not a comparison of actual observed changes, as the initial bathymetry is a modelled bathymetry as described in Section 5.2. The comparison shows where the assimilation system should produce changes to the initial bathymetry in order to match the final LiDAR data. The LiDAR data is subtracted from the initial model estimated bathymetry, so areas appearing positive (red and orange) are where erosion has occurred, and the initial estimate is higher than the LiDAR data; conversely, the blue and purple areas show where there has been deposition over the model run period. The main changes are in the migration of the Ulverston Channel, which shows as the red and orange area between the Leven and Kent estuaries (A), and the purple areas to the south of this (B). Along the Kent estuary there has been considerable deposition, which extends across to Wharton Sands (C). The Lune Estuary (D) is also higher as measured by the LiDAR data, while the channels leading up to Wharton Sands are deeper $(E)$. The differences between the initial bathymetry and the final model bathymetry produced by the assimilation scheme are shown in Fig. $9(\mathrm{~b})$ for comparison, using the values of $F=2.0, l=1.0$. If the assimilation system was perfect, the two figures would be identical. Although the two figures show changes occurring in similar areas, the results from the assimilation scheme do not show the larger areas of deposition along the Cartmel Wharf $(\mathrm{A})$, and the removal of sediment along the Ulverston Channel has been over-estimated. The changes in the Lune Estuary are also not apparent in the assimilation system. This may be in part because the model itself is simplistic and is not reflecting fully the sediment transport processes active in the bay, particularly secondary currents which are responsible for meander migration. This means that changes in the channel positions are not well 
represented by the model alone. The use of the waterline assimilation should correct for this in part, but can only do so if the changes are adequately captured by the waterline data. Also the temporal spacing of the data assimilated can be large in some cases, and is certainly not regular. In some cases waterlines are separated only by a matter of hours, in others several months elapse between observations. Where the model runs without assimilation for a period of months, it may drift away from the correct bathymetry. If the next assimilation is a waterline that does not cover the areas where the change has been greatest, it may not be able to correct the model sufficiently over the required areas, leaving discrepancies between the true state of the bathymetry and the prediction from the assimilation scheme.

(Figure 9)

\subsection{The impact of storms}

Storms in Morecambe Bay may be expected to affect the bathymetry due to storm surges and increased wave action. This can result in more rapid changes in the bathymetry during a storm, and would result in the model being less effective at predicting these changes, as it includes neither storm surges nor wave action. If this assumption is correct, the assimilation system would be expected to provide a larger correction to the predicted bathymetry after a storm than after a relatively calm period. As the observations are relatively sparse both temporally and spatially, it was not possible to find individual observations following each individual storm over the study period from January 2004 November 2005. Given this limitation, the approach taken was to divide the 23 month period into 'stormy' and 'quiet' periods. These periods were defined by looking at the wind speeds measured at Walney Island Observatory which provided the mean hourly wind magnitude and direction. A 'stormy period' was defined as one in which the wind reached $30 \mathrm{kts}$, with winds over $20 \mathrm{kts}$ for more than $5 \%$ of the time, and quiescent periods those in which the wind speeds rarely reached $20 \mathrm{kts}$. This gave two stormy periods, from January 2004 - April 2004, and October 2004 - March 2005. The quiescent periods were from May 2004 - September 2004, and April 2005 - November 2005. The bathymetry predicted by the assimilation system (using values of $F=2.0, \ell=1.0$ ) just before each waterline was assimilated was compared with the observations in that waterline and the variance of the differences calculated (Table 6). The two stormy and quiescent periods had differing numbers of waterlines in them, so a weighted average was taken for each period. The combined variance for the two stormy periods, $\sigma_{\mathrm{s}}$ was then compared with the variance for the quiet periods, $\sigma_{\mathrm{q}}$, using an F-test, where

$$
f_{t}=\sigma_{s} / \sigma_{q}
$$

If $f_{t}$ is significantly greater than 1 , the signal from the stormy periods can be seen; if the result of the Ftest is close to 1 , then there is no significant difference. The calculated value of $\sigma_{\mathrm{s}}$ was 1.485 , and that for $\sigma_{\mathrm{q}}$ was 1.284 , giving a value of 1.157 for the $\mathrm{F}$-test. This value suggests that the signal of greater variance in the stormy periods was not significantly detectable from the test we were able to perform. However, given that the intervals between the storms and the next assimilated data were variable (from 28 days to 55 days), and that the storms in each season were variable in strength, duration, and frequency, it is difficult to draw firm conclusions from this initial analysis. If data was available shortly after each storm, and at regular intervals during more quiescent periods, it might be possible to conduct a more accurate investigation of the ability of the assimilation system to aid the model recovery after individual storms, or after a stormy period.

\begin{tabular}{|cc|cc|}
\hline \multicolumn{4}{|l|}{$\begin{array}{l}\text { Table 6 } \\
\text { Variances for stormy (s1 (January 2004 - April 2004) and s2 } \\
\text { (October 2004 - March 2005)) and quiescent (q1 (May 2004- } \\
\text { September 2004) and q2 (April }\end{array}$ 2005 - November 2005)) periods $\left(\mathrm{m}^{2}\right)$} \\
\hline $\mathrm{s} 1$ & $\mathrm{~s} 2$ & $\mathrm{q} 1$ & $\mathrm{q} 2$ \\
\hline 2.13 & 1.72 & 2.61 & 1.68 \\
\hline 1.04 & 2.06 & 0.45 & 0.62 \\
\hline 0.43 & & 1.29 & 1.02 \\
\hline 1.53 & & & 1.32 \\
\hline
\end{tabular}




\begin{tabular}{|c|c|c|}
\hline $\begin{array}{c}\text { Overall } \\
\text { average }\end{array}$ & 1.485 & 1.284 \\
\hline
\end{tabular}

\section{Comparison of Optimal Interpolation and 3D-Var}

The Optimal Interpolation (O.I) method as presented in Scott and Mason (2007) was also tested, with the same value of $\sigma_{\mathrm{b}}{ }^{2}$, and a similar range of $\mathrm{F}$ and $\ell$ as that used in the 3D-Var method. It included the same set of waterline data and the swath bathymetry in the assimilation scheme. The main difference in the two methods is in the way that the B matrix is defined: in the O.I. method the B matrix can be specified directly, and is based on a Gaussian approximation for the error covariances. The 3D-Var method uses a Laplacian approximation, as described in section 5.3. The O.I. method uses one scalar analysis equation for each model cell, and uses a different subset of the observations for each cell. The 3D-Var method minimizes the cost function for the entire domain, using all the available observations.

For this comparison, the parameter ranges used were the same as for the 3D-Var version, and the broad equivalence of the $\mathbf{B}$ matrices was assumed. The $\mathbf{R}$ matrix was the same in both cases, as the observations used were identical. The Brier skill scores calculated against the swath dataset for the two types of assimilation method are shown in Fig. 10 for various values of $F$, following the method used for the calibration discussed in section 6 . This shows that in both cases, the optimum value for $F$ is 2.0 , and also that the 3D Var method results in slightly higher values for the Brier skill score. When the results after 23 months are compared with the LiDAR data and the skill score calculated using this dataset (Fig. 11), the optimum value for $F$ is 1.0 for both the O.I. and 3D Var cases. The scores are almost identical for the lower values of $F$, but the O.I. method shows an improvement over the 3D Var for the higher values. The differences in the two methods do not have a large impact on the final predicted bathymetry, suggesting that the differing implementations for the background error covariance matrix $B$ have a similar effect on the assimilation of the observations.

(Figure 10) (Figure 11)

\section{Conclusions}

The application of the 3D-Var assimilation method to the morphodynamic modelling of Morecambe Bay has demonstrated that using data assimilation improves the outcome of the simple morphodynamic model substantially over the model run period of 23 months. The system is calibrated, and then validated against an independent dataset. The approximation of the error covariance matrix for the model has also been calibrated to find the best length scale for the system, and produces results that optimize the use of the data without extending the information that the data provides beyond its usefulness. Combining the range of values for the sediment transport model and the length scale to produce a form of ensemble improves the overall match of the assimilation system compared with using individual runs. Future investigation might consider whether using variable values for $F$ and $\ell$ in an individual run might also improve the overall match with the LiDAR data.

The Laplacian approximation for the $\mathbf{B}^{-1}$ matrix works well, but other structures for the error covariance method could be investigated, and adaptation over time could also be considered.

The difference between the 3D-Var and O.I. methods is not dramatic, and the comparison is not direct because of the differences in the definition of the model error covariance matrix. However, the greater flexibility of the 3D-Var system and its amenability for including data from different sources and the option of expanding the system to include parameter estimation make this a better choice overall for the assimilation method.

The utility of assimilation in improving the prediction for bathymetry can provide a useful alternative to either expensive and frequent surveys of the coastal bathymetry, or the development of complex and computationally intensive models. However, using a model that represents more of the physical processes operating than in the work presented here would also improve the outcomes, provided that a suitable way of defining the model errors could be found. Further investigation into the optimal frequency and spatial coverage of the observations would lead to a more efficient use of surveys and remote sensing imagery for future assimilation systems. The effect of the sparse spatial and temporal coverage of the data, and examining the options for concentrating data gathering over the most dynamically evolving areas (e.g. channels) may improve the efficiency of this type of assimilation system. For risk assessment the areas considered most vulnerable to flooding and most likely to be affected by changes in the bathymetry could provide a focus for future observations. The combination of satellite data such as the SAR imagery, LiDAR from airborne platforms, and local in situ surveys 
could all be incorporated into a simple model such as the one described here, providing considerable improvements in the ability to predict changes in bathymetry in Morecambe Bay.

\section{Acknowledgements}

We would like to thank Lancaster City Council for providing the swath bathymetry and the LiDAR data used in this work.

This work was funded under the NERC Flood Risks from Extreme Events (FREE) programme (Grant no NE/E002048/1).

\section{References}

Bannister, R. N., 2008. A review of forecast error covariance statistics in atmospheric variational data assimilation. I: Characteristics and measurements of forecast error covariances. Q. J. R. Meterol. Soc. 134, 1951-1070.

Bouttier, F., Courtier, P. 1999. Data assimilation concepts and methods. http://www.ecmwf.int/newsevents/training/rcourse_notes/DATA_ASSIMILATION/ASSIM_CONCEPTS/ Assim_concepts21.html.

Camenen, B., Larroude, P., 2003. Comparison of sediment transport formulae for the coastal environment. Coastal Engineering 48, 111-132.

Coomber, D.P.M., Hansom, J.D., 1994. Estuaries Management Plans: Coastal Processes and Conservation, Morecambe Bay. Coastal Research Group, University of Glasgow, 79pp.

de Vriend, H.J., 1987. 2DH mathematical modelling of morphological evolutions in shallow water. Coastal Engineering 11, 1-27.

de Vriend, H.J., Zyserman, J., Nicholson, J., Roelvink, J. A., Pechon, P., Southgate, H. N. 1993. Medium-term 2DH coastal area modelling. Coastal Engineering 21, 193-224.

Järvinen, H. 1998. Observations and diagnostic tools for data assimilation, http://www.ecmwf.int/newsevents/training/rcourse_notes/DATA_ASSIMILATION/OBS_AND_DIAG_TO OLS/Obs_and_diag_tools.html

Johnson, C., 2003. Informational content of observations in variational data assimilation. PhD Thesis, Department of Mathematics, University of Reading.

Johnson, C., Hoskins, B.J., Nichols, N. K., 2005. A singular vector perspective of 4D-Var: Filtering and interpolation. Quarterly Journal of the Royal meteorological Society, 131 part A, 1, 19.

Laub, C., Kuhl, T. L., 2005. How Bad is Good? A Critical Look at Iterative Fitting of Reflectivity Models using the Reduced Chi-Square Statistic. SNS - HFIR Users Meeting 2005, Oak Ridge National Laboratory, Oak Ridge, TN, USA.

Lesser, G. R., Roelvink, J. A., van Kester, J. A. T. M., Stelling, G. S., 2004.Development and validation of a three-dimensional morphological model. Coastal Engineering 51, 883-915.

Lorenc, A. C., 1986. Analysis methods for numerical weather prediction. Quat. J. R. Met. Soc, 112, 1177-1194.

Mason, D. C., Amin, M., Davenport, I. J., Flather, R.A., Robinson, G.J., Smith, J. A., 1999.

Measurement of recent intertidal sediment transport in Morecambe Bay using the waterline method. Estuarine, Coastal and Shelf Science 49, 427-456. 
Mason, D.C., and Garg, P.K., 2001. Morphodynamic Modelling of Intertidal Sediment Transport in Morecambe Bay. Estuarine, Coastal and Shelf Science, 53, 79-52.

Mason DC, Scott TR and Dance SL (2010). Remote sensing of intertidal morphological change in Morecambe Bay, U.K., between 1991 and 2007. Estuarine Coastal and Shelf Science, 87, 487-496.

Scott, T.R., Mason, D.C., 2007. Data assimilation for a coastal area morphodynamic model:

Morecambe Bay. Coastal Engineering 54, Issue 2, 91-109.

Shanno, D. F., Phua, K. H., 1980. Algorithm 500: Minimization of Unconstrained Multivariate Functions [E4]. ACM Transactions on Mathematical Software (TOMS). 6(4), 618 - 622.

Shoreline Management Partnership (SMP), 1996. Morecambe Bay Shoreline Management Plan (Consultation Document) Stage 1 Volume 2. Report to the Cumbria Coastal Consortium.

Smith, P. J., Dance, S. L., Baines, M. J., Nichols. N. K., and Scott, T. R., 2009, Variational data assimilation for parameter estimation: application to a simple morphodynamic model. Ocean Dynamics Vol 59, No. 5, 697-708.

Soulsby, R. 1997. Dynamics of Marine Sands. Thomas Telford Publications, London.

Stewart, L.M., Dance, S.L. and Nichols, N.K. Correlated observation errors in data assimilation. International Journal for Numerical Methods in Fluids, 56(8), 2008, 1521-1527.

Stewart, L. M., 2010. Correlated observation errors in data assimilation. PhD Thesis, Department of Mathematics, University of Reading.

Sutherland, J., Peet, A. H., Soulsby, R.L. 2004a. Evaluating the performance of morphological models. Coastal Engineering 51, 917-939

Sutherland, J., Walstra, D. J. R., Chesher, T. J., van Rijn, L. C., Southgate, H. N., 2004b. Evaluating the performance of morphological models. Coastal Engineering 51, 917-939.

Van Dongeren A, Plant N, Cohen A, Roelvink D, Haller MC, Catalan PA (2008). Beach Wizard: Nearshore bathymetry estimation through assimilation of model computations and remote observations. Coastal Engineering 55(12), 1016-1027.

Woodcock, F., Engel, C., 2005. Operational Consensus Forecasts. Weather and Forecasting, 20, 101 111. 
Fig. 1: Main geographic features of Morecambe Bay (based on O.S. maps (revised 1968-71)

Fig. 2: Datasets: a) Waterline for $30^{\text {th }}$ January 2004. b) Swath bathymetry coverage, shown as black lines. The underlying bathymetry shown is the initial bathymetry used for the model runs. c) LiDAR data for November 2005

Fig. 3: (a) Bathymetry for 1997 (after Mason et al 1999) (b) Initial bathymetry created as initial bathymetry for January 2004

Fig. 4: Background - analysis showing the effect of different length scales: (a) $\ell=0.5$ (b) $\ell=3.0$

Fig. 5: Plots of differences in the bathymetry for ranges of parameters. The letters in each plot are referred to in the text.

a) Differences in predicted bathymetry between the maximum and minimum values of $\ell$ after 23 months. The plot shows the bathymetry for $\ell=3.0$ subtracted from the bathymetry for $\ell=0.5$. b) Differences in predicted bathymetry between the maximum and minimum values of $F$ after 23 months. The plot shows the bathymetry for $F=3.0$ subtracted from the bathymetry for $F=0.1$. c) Differences in predicted bathymetry between values of $\sigma_{b}^{2}=0.8$ and $\sigma_{b}{ }^{2}=2.0$ after 23 months. The plot shows the bathymetry for $\sigma_{b}{ }^{2}=2.0$ subtracted from the bathymetry for $\sigma_{\mathrm{b}}^{2}=0.8$.

Fig. 6: Plots of differences between predicted bathymetry and bathymetry measured by LiDAR (prediction - data). The letters in each plot are referred to in the text.

(a) without assimilation (b) with assimilation. c) Differences between model output bathymetry and LiDAR data for model run excluding assimilation of swath data. d) Differences between weighted ensemble mean bathymetry and LiDAR data.

Fig. 7: Graph of Brier skill score against time for runs with and without assimilating the swath data. The lines marked 'WL' mark the times of waterline data assimilations.

Fig. 8: Weighted standard deviation for all individual model runs used to construct the ensemble.

Fig. 9: Change in bathymetry over 23 months from initial model bathymetry: (a) compared to LiDAR data (b) compared to final model bathymetry prediction. The letters in each plot are referred to in the text.

Fig. 10: Comparison of Brier skill scores relative to swath observations for O.I. and 3D-Var assimilation methods

Fig. 11: Comparison of Brier skill scores relative to LiDAR observations for O.I. and 3D-Var assimilation methods 


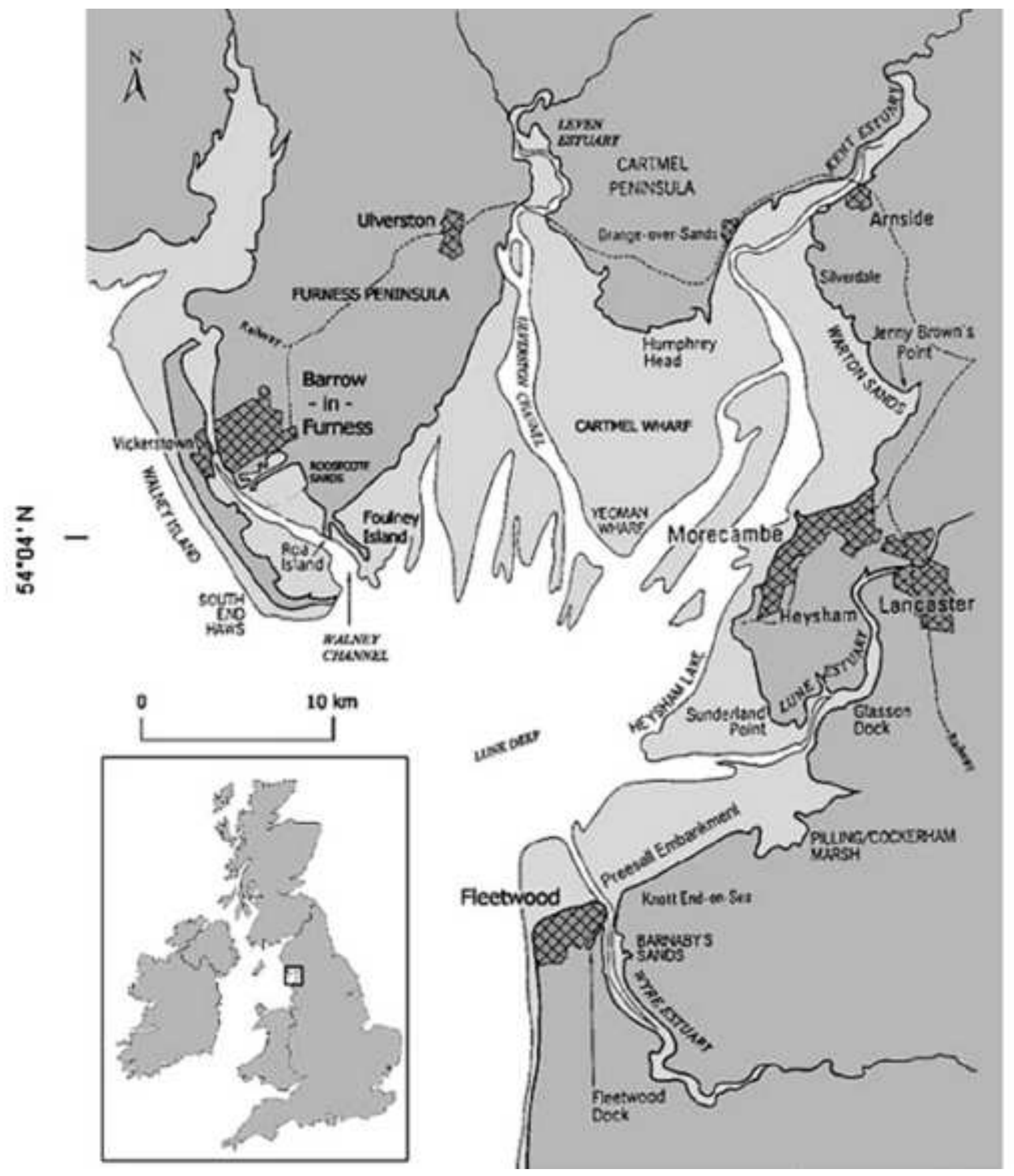


Click here to download high resolution image
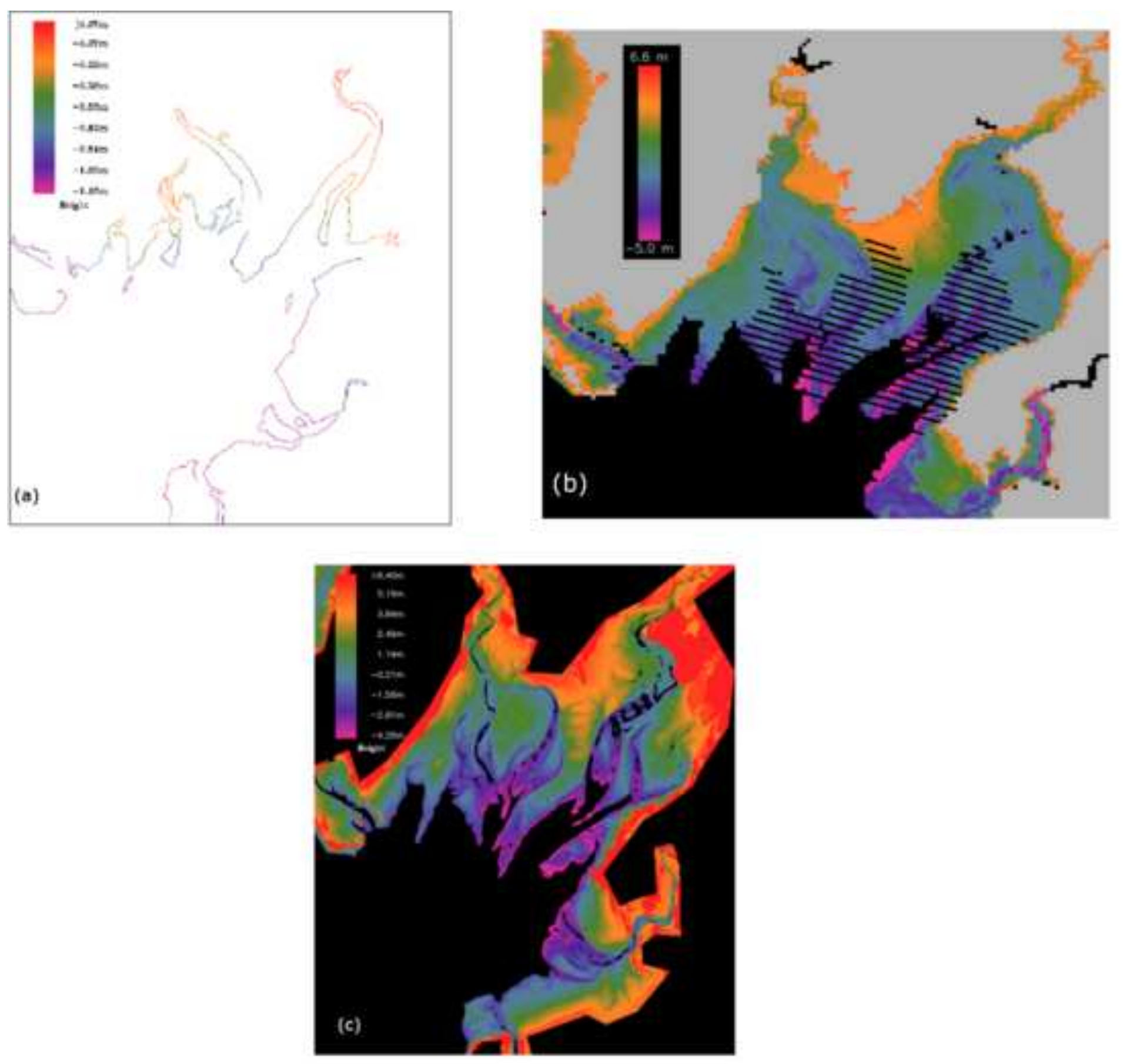
Click here to download high resolution image
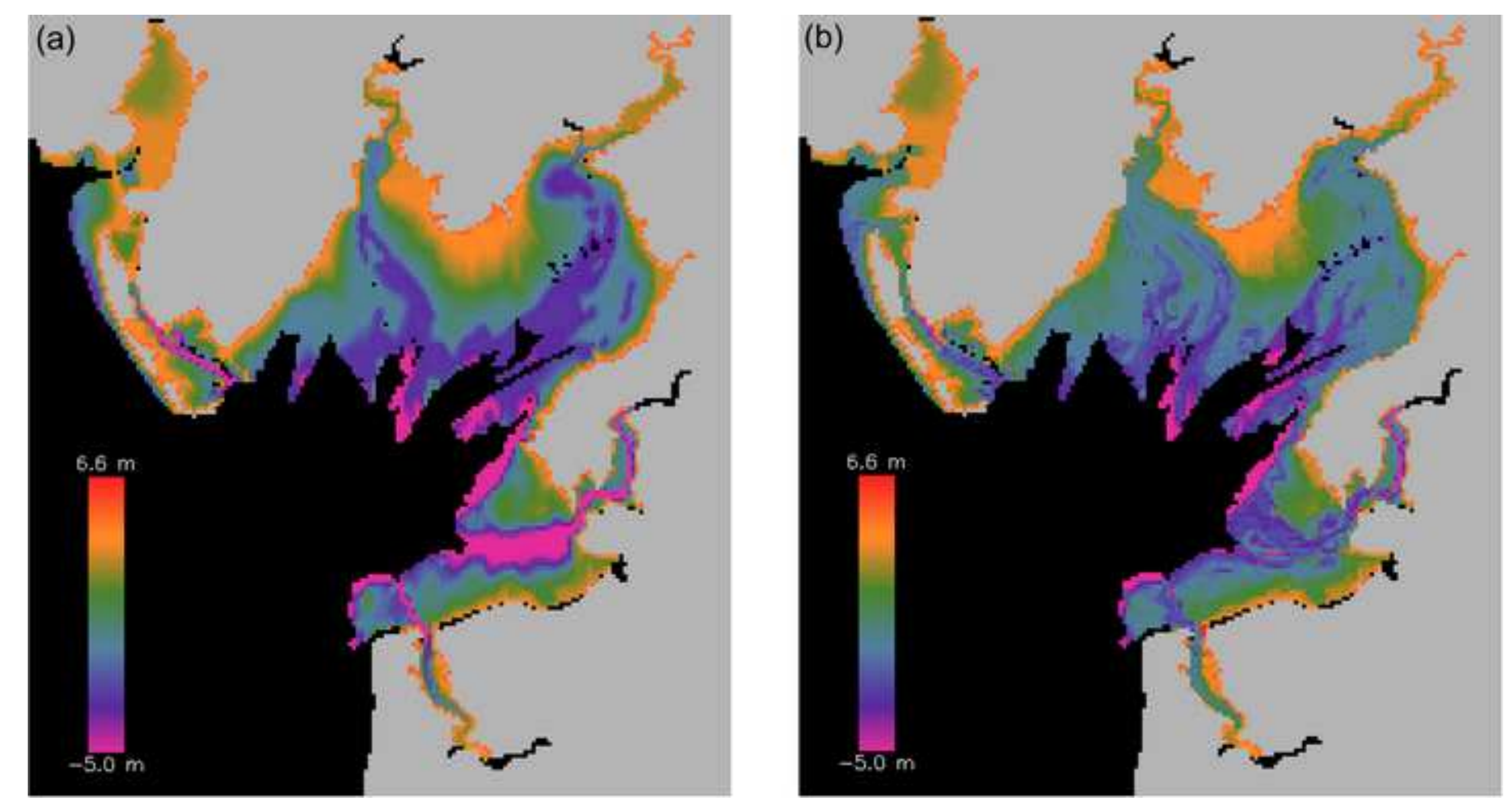

Click here to download high resolution image 

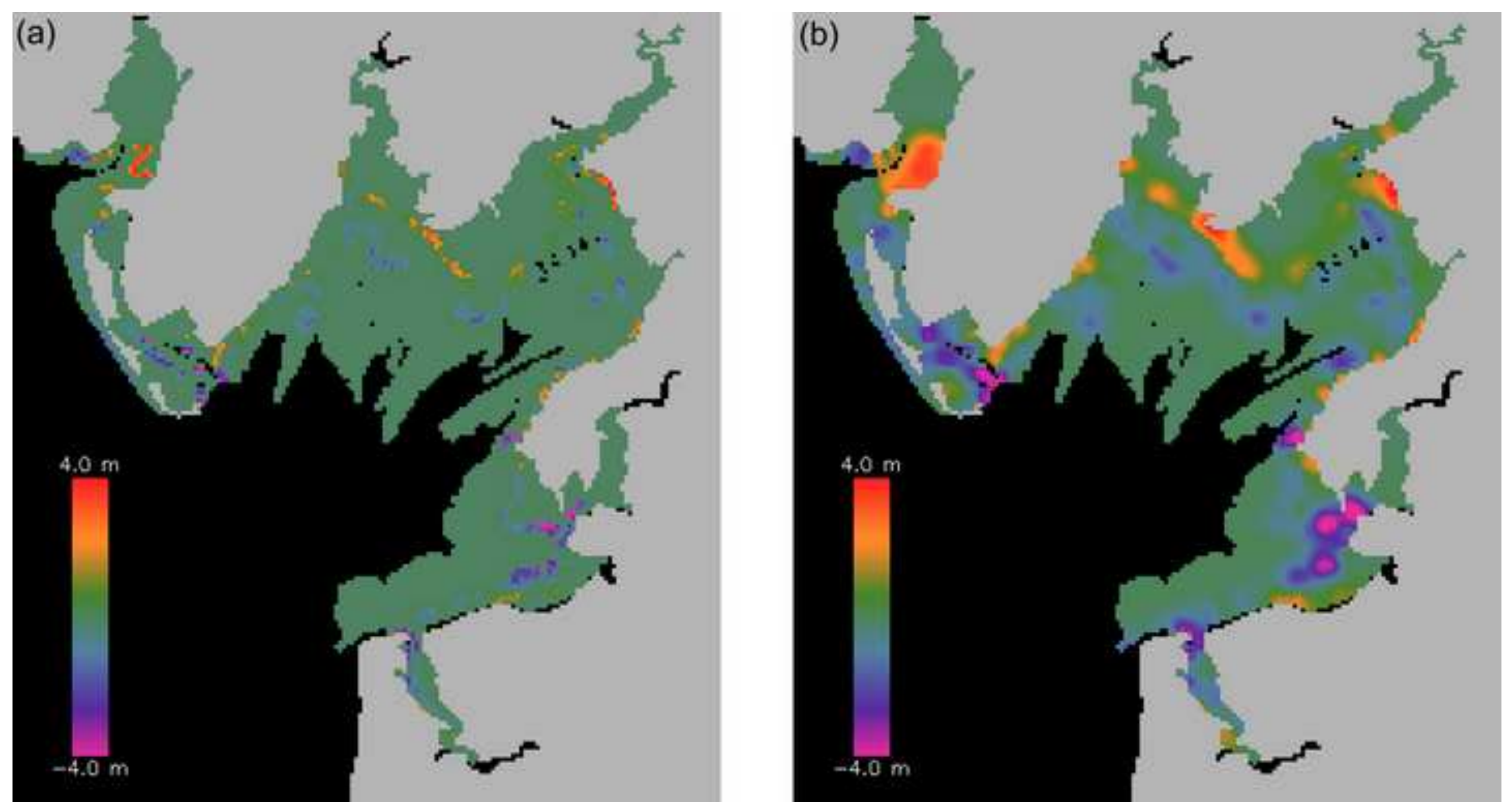
Click here to download high resolution image
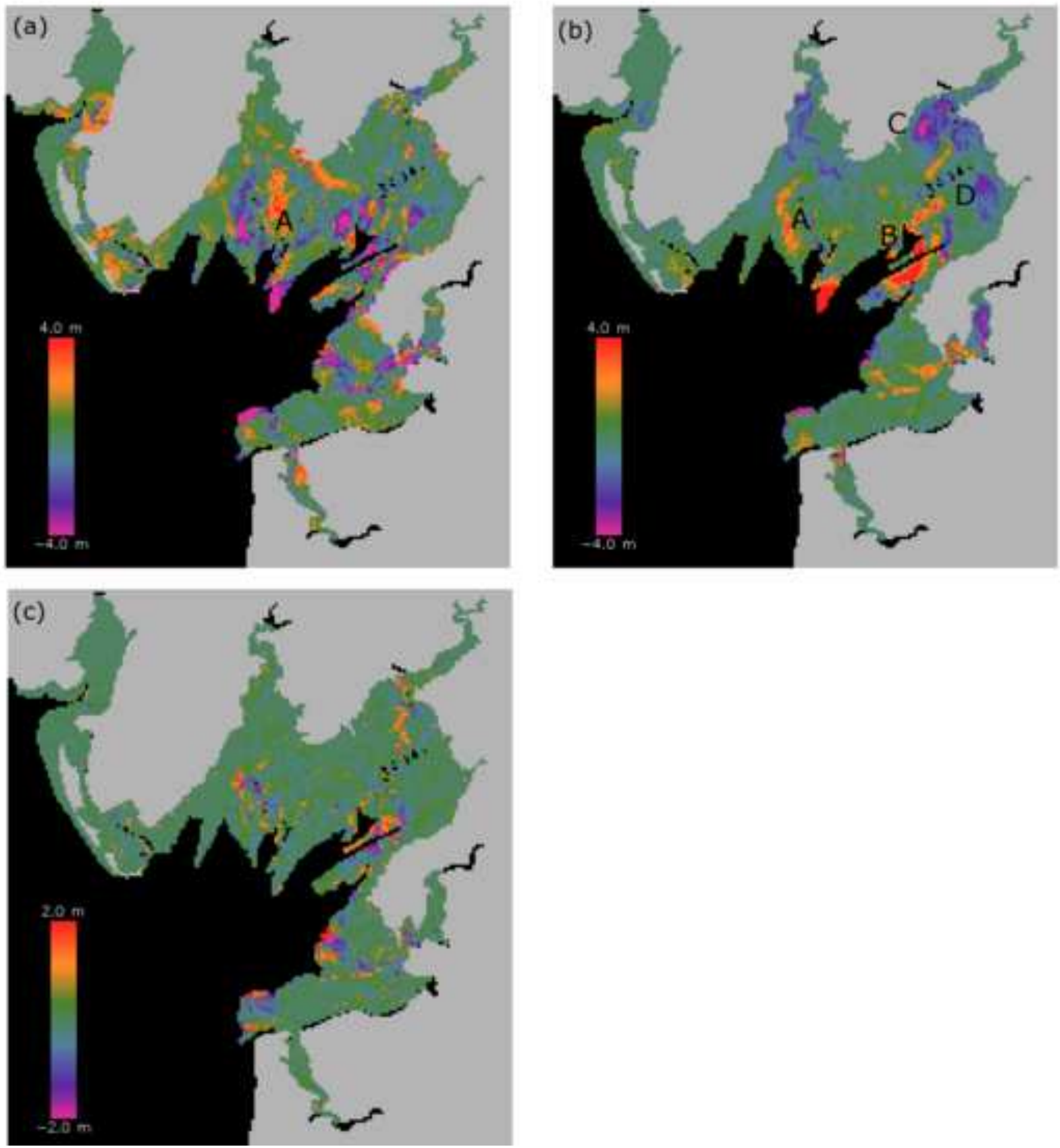
Click here to download high resolution image
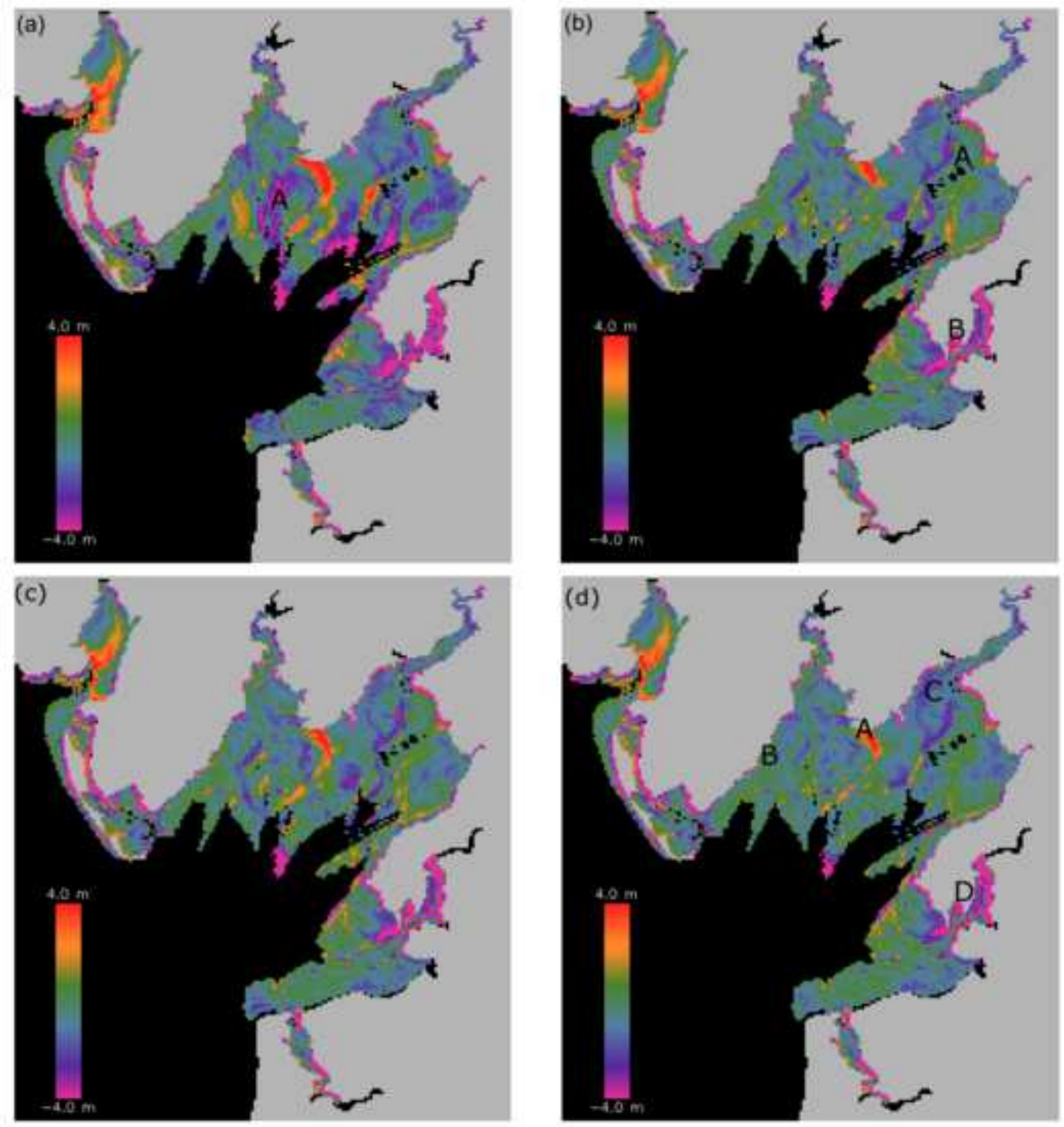
BSS with and without swath assimilation

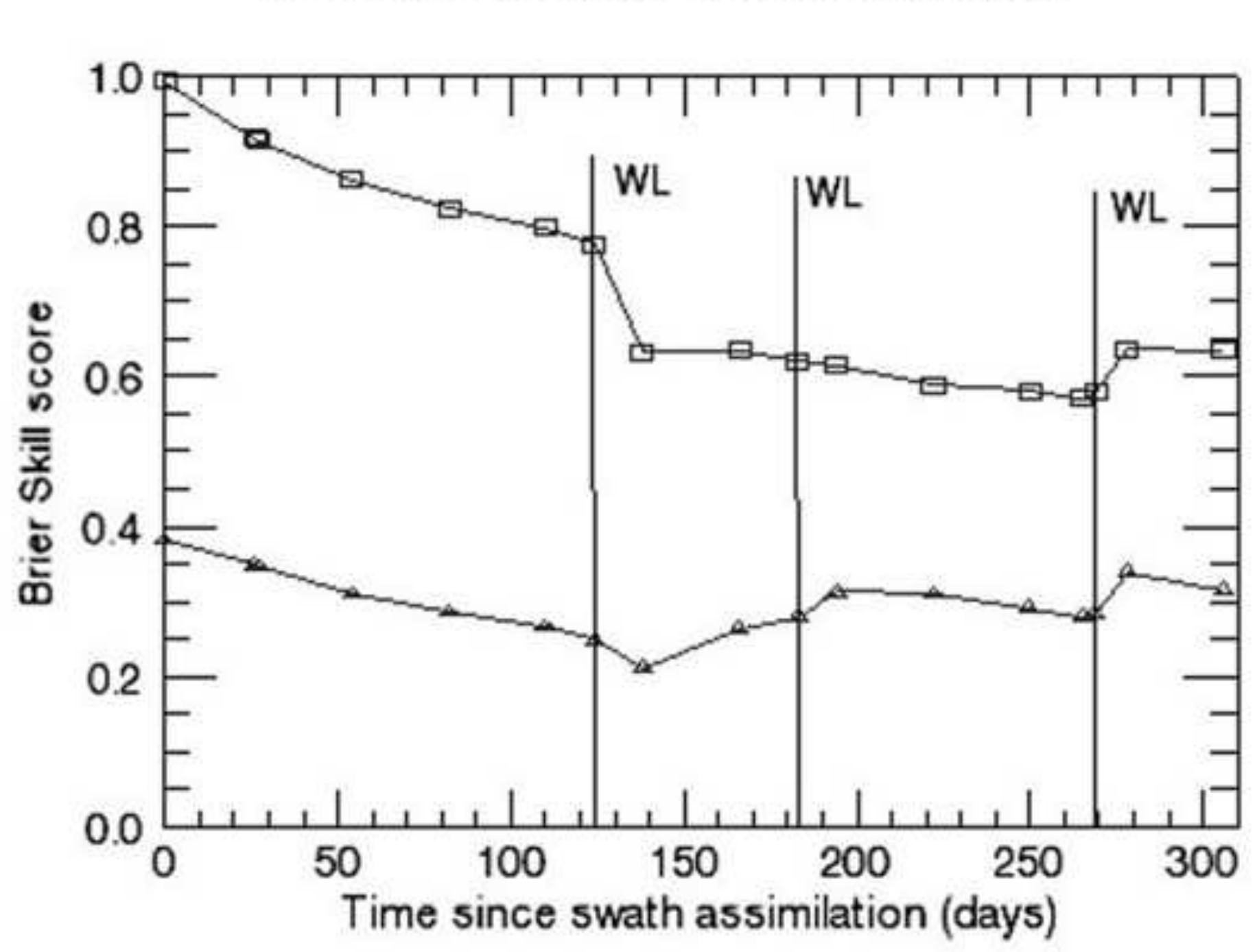

$$
\begin{aligned}
& \Delta-\triangle \text { Swath not assimilated } \\
& \square-\square \text { With swath assimilated }
\end{aligned}
$$


Click here to download high resolution image

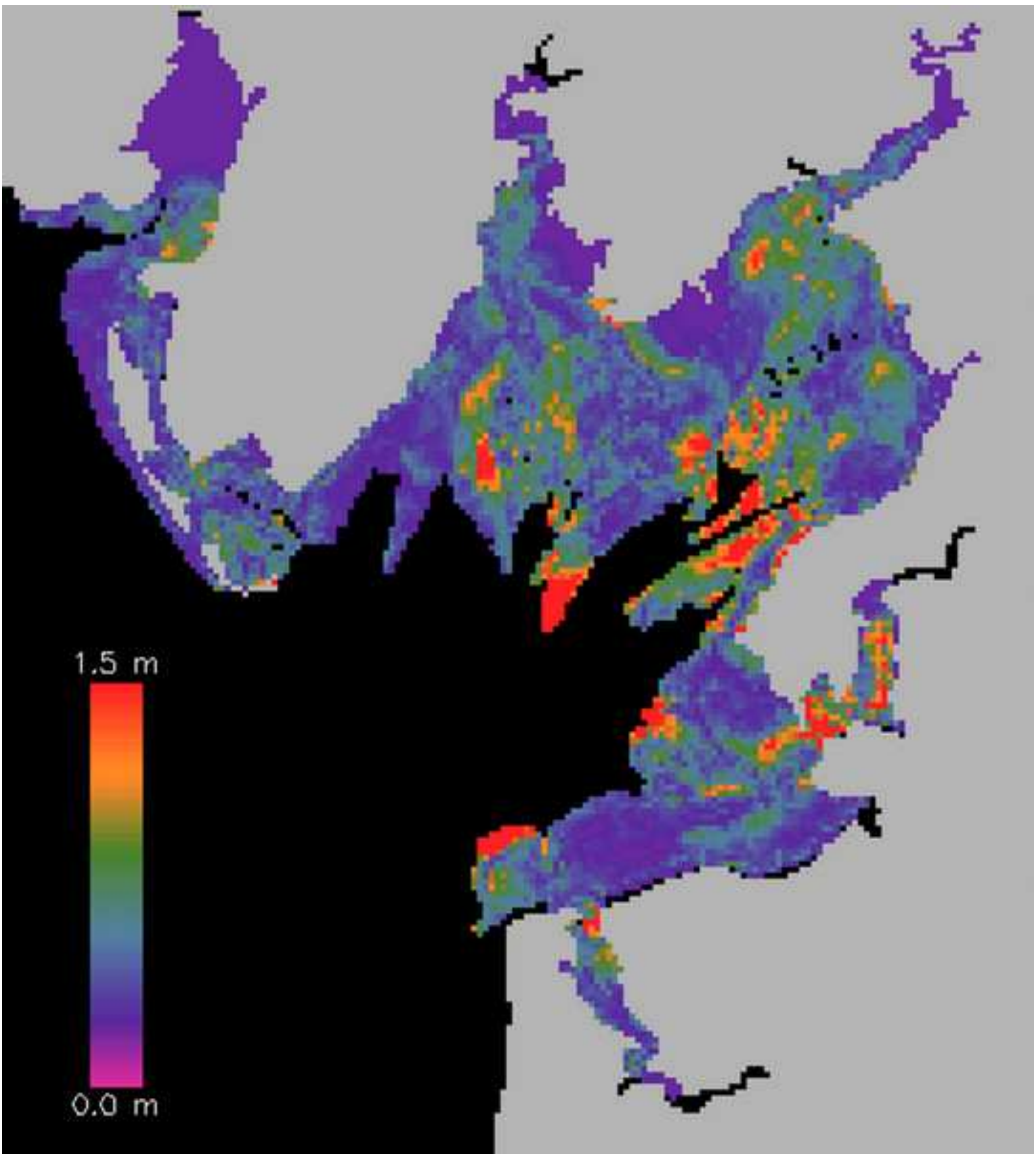



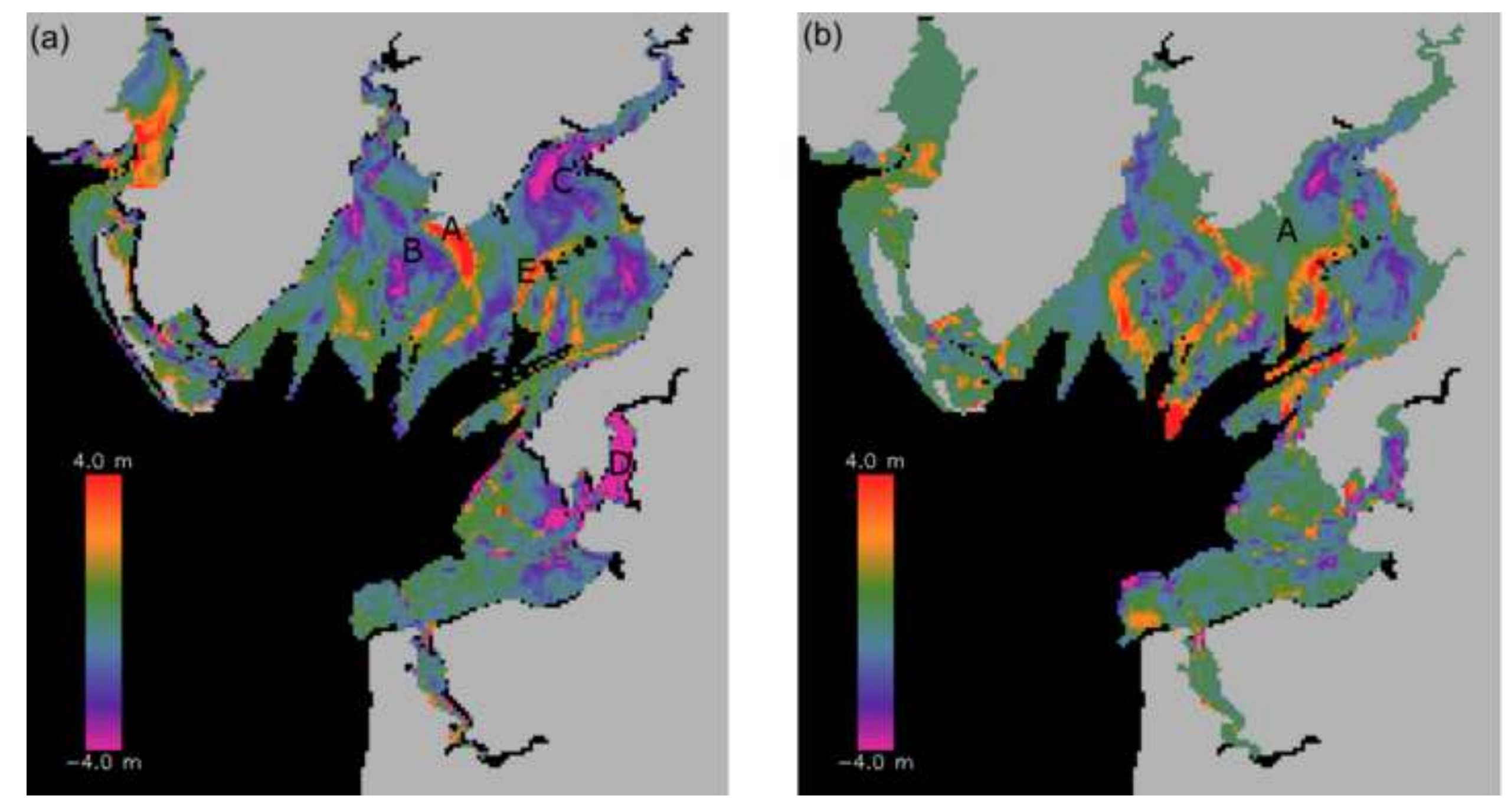

Click here to download high resolution image 
Comparison of O.I. and 3D Var using Swath data

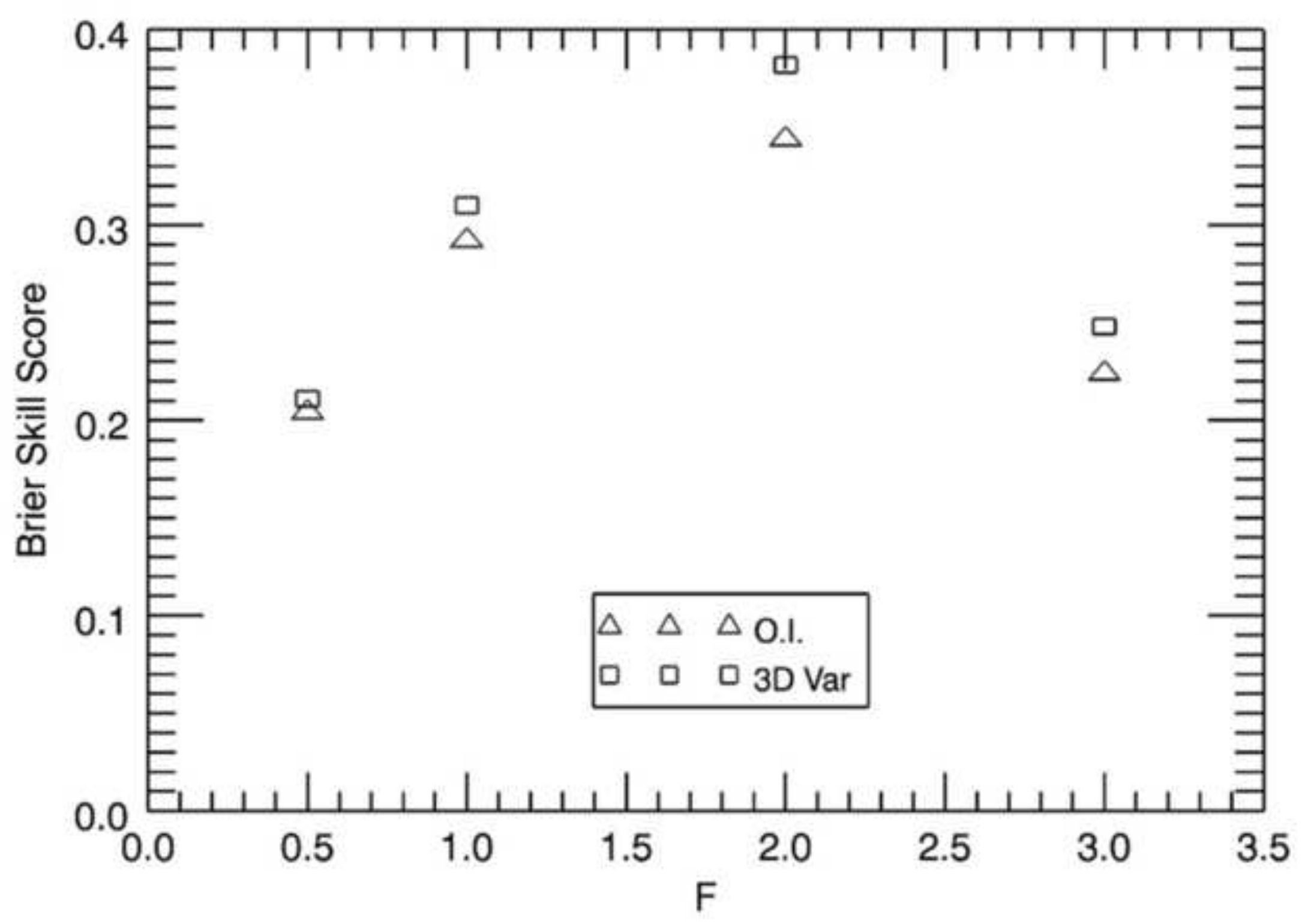


Comparison of O.I. and 3D Var using LiDAR data

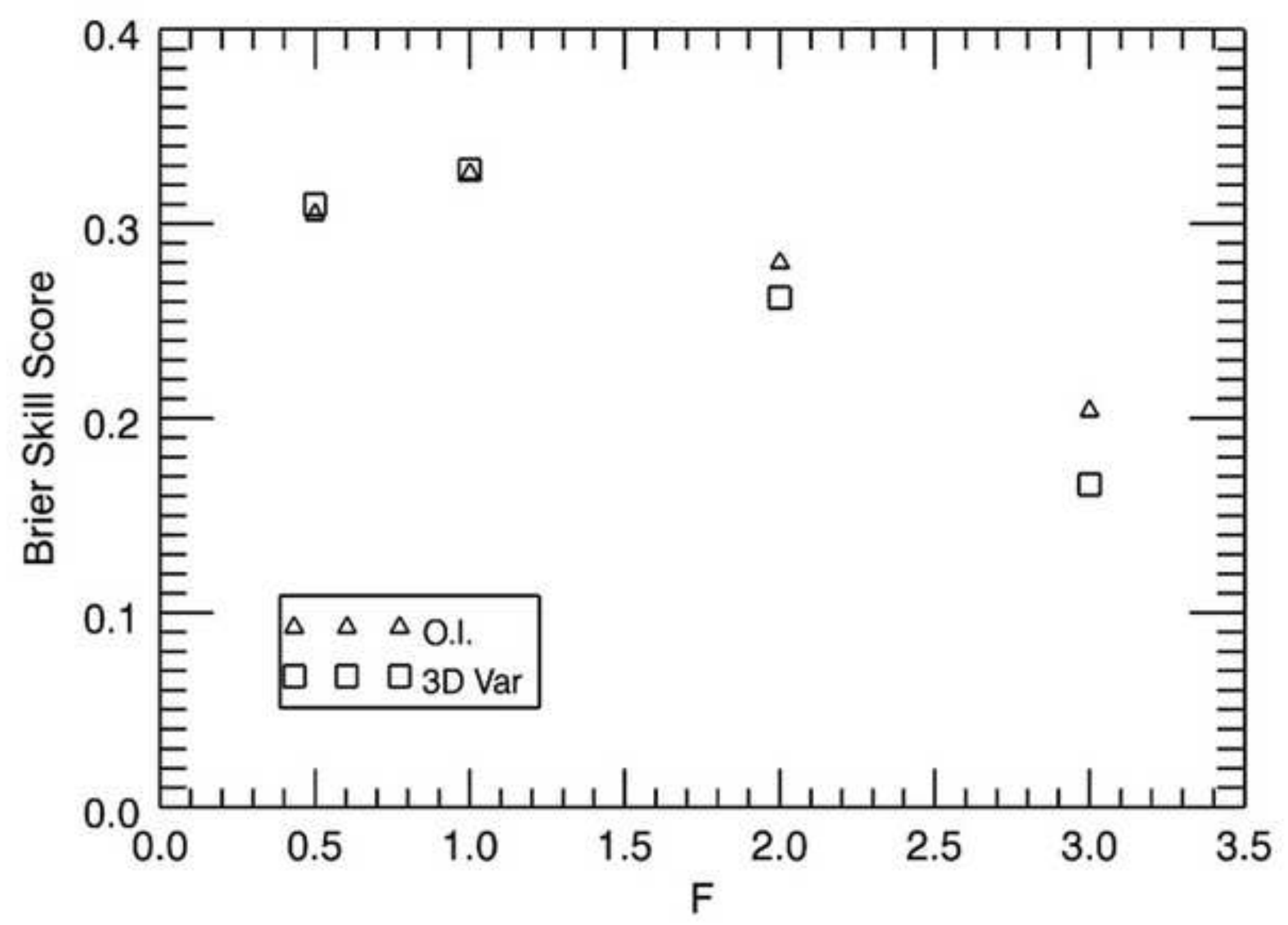

